\title{
ADAPTIVE FETI-DP AND BDDC METHODS WITH A GENERALIZED TRANSFORMATION OF BASIS FOR HETEROGENEOUS PROBLEMS*
}

\author{
AXEL KLAWONN $^{\dagger}$, MARTIN KÜHN ${ }^{\dagger}$, AND OLIVER RHEINBACH ${ }^{\ddagger}$
}

\begin{abstract}
In FETI-DP (Finite Element Tearing and Interconnecting) and BDDC (Balancing Domain Decomposition by Constraints) domain decomposition methods, the transformation-of-basis approach is used to improve the convergence by combining the local assembly with a change of basis. Suitable basis vectors can be constructed by the recently introduced adaptive coarse space approaches. The resulting FETI-DP and BDDC methods fulfill a condition number bound independent of heterogeneities in the problem. The adaptive method with a transformation of basis presented here builds on a recently introduced adaptive FETI-DP approach for elliptic problems in three dimensions and uses a coarse space constructed from solving small, local eigenvalue problems on closed faces and on a small number of edges. In contrast to our earlier work on adaptive FETI-DP, the coarse space correction is not implemented by using balancing (or deflation), which requires the use of an exact coarse space solver, but by using local transformations. This will make it simpler to extend the method to a large number of subdomains and large supercomputers. The recently established theory of a generalized transformation-of-basis approach yields a condition number estimate for the preconditioned operator that is independent of jumps of the coefficients across and inside subdomains when using the local adaptive constraints. It is shown that all results are also valid for BDDC. Numerical results are presented in three dimensions for FETI-DP and BDDC. We also provide a comparison of different scalings, i.e., deluxe, rho, stiffness, and multiplicity for our adaptive coarse space in 3D.
\end{abstract}

Key words. domain decomposition, FETI-DP, BDDC, coarse space, adaptive, eigenvalue problem, elliptic partial differential equations

AMS subject classifications. $65 \mathrm{~N} 30,65 \mathrm{~N} 55,65 \mathrm{~F} 08,65 \mathrm{~F} 10$

1. Introduction. Domain decomposition methods $[49,59,62]$ are widely-used iterative methods for the parallel solution of implicit finite element problems. In these methods, the finite element problem is decomposed into parallel local problems. Additionally, to obtain scalability in the number of iterations, a coarse space ensures the global transport of information.

Originally, the coarse space of FETI-DP and BDDC methods [11, 13, 17, 18, 19, 62] is formed by coupling the subdomains in a few primal variables, i.e., a partial finite element assembly is used to enforce continuity across subdomain boundaries in a few degrees of freedom such as corner nodes.

In three dimensions, this is not sufficient to obtain a good condition number bound, and, e.g., continuous edge averages have to be enforced throughout the iteration additionally. A transformation of basis to explicitly introduce the averages as new variables and a subsequent partial finite element assembly is a technique to implement such average constraints in FETI-DP and BDDC methods; see, e.g., [35, 40, 41, 45]. An alternative is the use of the edge characteristic functions as deflation vectors in a deflation (also known as projector preconditioning) or balancing approach; see, e.g., [38]. Indeed, for different problems, many different FETI-DP and BDDC coarse spaces have been developed over time.

It has been shown in $[24,38]$ for homogeneous problems that for any FETI-DP method with a transformation of basis there exists a FETI-DP method using deflation or balancing with essentially the same spectrum [38, Theorem 6.7]. In [30], we have shown that for heterogeneous problems, a generalized transformation-of-basis approach is necessary when

\footnotetext{
* Received July 26, 2017. Accepted January 11, 2018. Published online on March 5, 2018. Recommended by Marcus Sarkis.

${ }^{\dagger}$ Universität zu Köln, Mathematisches Institut, Weyertal 86-90, 50931 Köln, Germany

( $\{$ axel.klawonn, martin.kuehn\}@uni-koeln.de).

${ }^{\ddagger}$ Technische Universität Bergakademie Freiberg, Fakultät für Mathematik und Informatik, Institut für Numerische Mathematik und Optimierung, 09596 Freiberg, Germany (oliver.rheinbach@math.tu-freiberg. de).
} 
a second coarse space is introduced to improve a domain decomposition method with an initial (a priori) coarse space and a corresponding scaling. Such a posteriori constraints, i.e., constraints that are computed after the choice of an initial coarse space and a scaling, are typical for domain decomposition methods with automatic or so-called adaptive coarse spaces. These approaches have been introduced for highly heterogeneous problems, notably in compressible or almost incompressible elasticity with coefficient jumps. For the resulting coarse spaces the condition number then is independent of the heterogeneities. Of course, this comes at the price of a larger coarse problem. It is therefore important that an automatic coarse space generates a small a posteriori coarse space.

The adaptive (i.e., problem-specific) coarse spaces are constructed automatically, i.e., during the computation typically by (approximately) solving local eigenvalue problems. Since these computations are performed after the introduction of the scaling, the constraints are scaling dependent, and the enforcement of these constraints has to be carried out by a generalization of the transformation-of-basis approach as introduced in [30]. A subtle detail of this new method, which is relevant for the theory, is that we observe nontrivial values at coarse nodes of the a posteriori or second coarse space when the FETI-DP or BDDC operators $P_{D}$ or $I-E_{D}$ are applied to functions from the new constraint space; cf. [30].

Recently, such domain decomposition methods with adaptive coarse spaces have gained considerable interest. In [6, 7], adaptive Neumann-Neumann methods were considered. Adaptive Schwarz methods were considered later by [15, 16, 20, 21, 60], where one of these methods was generalized to adaptive BDD and FETI; see [61].

For FETI-DP and BDDC, adaptive methods were introduced or considered in [5, 9, 12, 26, 27, 28, 31, 33, 34, 47, 48, 52, 53]. In adaptive FETI-DP and BDDC methods, typically (approximate) eigenvectors from local eigenvalue problems on, e.g., faces or edges, are integrated into the coarse space. A deflation or balancing approach, as in [38], can then be used to implement the coarse space correction using the eigenvectors as deflation vectors. This approach can be used with different automatic coarse space strategies and was used in [33] for a comparison of adaptive approaches in two dimensions. As shown by the authors in [28], deflation can also be used in three dimensions to create an adaptive FETI-DP method with a condition number bound for heterogeneous problems that is independent of the jumps of the coefficients. The approach in [28] thus sets the successful heuristics introduced in [47], using only face eigenvalue problems, on a firm theoretical ground by adding a small number of edge eigenvalue problems.

However, in the deflation and balancing approach as used in [28], the coarse correction has to be computed quite exactly to ensure convergence to the correct solution; see [38]. When the coarse problem becomes large, an exact solution of the coarse problem can be inefficient.

Using a transformation-of-basis (or change-of-variables) approach combined with partial assembly can be an efficient alternative; see also, e.g., [35, 40]. Building on this, highly scalable inexact FETI-DP methods have been constructed as in $[32,36]$ that scale to hundreds of thousands of processor cores using a single cycle of an AMG method for the coarse problem.

In this current paper, we show that for adaptive coarse spaces, the generalized transformation-of-basis approach [30] can be used instead of deflation as in [28]. As in [28], we obtain a condition number bound independent of heterogeneities. This paper thus combines the generalized transformation-of-basis approach for FETI-DP with the adaptive coarse space [28] for problems in three dimensions and gives a condition number bound for this new approach. We will argue that the explicit condition number of the adaptive FETI-DP or BDDC method with the generalized transformation-of-basis approach is always smaller or equal to the condition number of FETI-DP with balancing or deflation. 
Adaptive coarse spaces for BDDC methods, e.g., [5, 9, 52, 53], are related to our approach, and we will also give a condition number bound and present numerical results for the coarse space of [28] with BDDC using the generalized transformation-of-basis approach. This extends [28], where only FETI-DP was considered because deflation for FETI-DP and BDDC are not equivalent [38].

The remainder of the paper is organized as follows. In Section 2 we introduce the model problem considered and the geometry used. In Section 3, we shortly rephrase the standard FETI-DP and BDDC methods. In Section 4, we introduce the generalized eigenvalue problems already considered in [28] that can result in adaptive constraints for FETI-DP and BDDC enforceable by the generalized transformation of basis as presented in [30]. This section will be concluded by a condition number bound for both the adaptive FETI-DP and the adaptive BDDC method. A vast overview on the numerical behavior of the new algorithm and of heuristic modifications thereof (cf. [28]) will be presented in Section 5. In Section 6, we provide some preliminary results using a first parallel implementation of our adaptive FETI-DP methods. Finally, we draw a conclusion in Section 7.

2. Model problem and geometry. Given a bounded polyhedral domain $\Omega \subset \mathbb{R}^{3}$ with $\partial \Omega_{D} \subset \partial \Omega$ a closed subset of positive surface measure and $\partial \Omega_{N}:=\partial \Omega \backslash \partial \Omega_{D}$, we consider the weak formulation of (compressible) linear elasticity. We introduce the space $H_{0}^{1}\left(\Omega, \partial \Omega_{D}\right)^{3}:=\left\{v \in H^{1}(\Omega)^{3}: v=0\right.$ on $\left.\partial \Omega_{D}\right\}$ as well as the Young Modulus $\mathrm{E}(x)>0$ and the Poisson ratio $0<\nu(x)<1 / 2$ for all $x \in \Omega$, and we are interested in finding $u \in H_{0}^{1}\left(\Omega, \partial \Omega_{D}\right)^{3}$ such that

$$
a(u, v)=F(v) \quad \forall v \in H_{0}^{1}\left(\Omega, \partial \Omega_{D}\right)^{3},
$$

where

$$
\begin{aligned}
a(u, v) & :=\int_{\Omega} 2 \mu \varepsilon(u): \varepsilon(v) \mathrm{d} x+\int_{\Omega} \lambda \operatorname{div}(u) \operatorname{div}(v) \mathrm{d} x, \\
F(v) & :=\int_{\Omega} f \cdot v \mathrm{~d} x+\int_{\partial \Omega_{N}} g \cdot v \mathrm{~d} s .
\end{aligned}
$$

Here, $\lambda$ and $\mu$ are the Lamé constants, and

$$
\varepsilon(u): \varepsilon(v)=\operatorname{tr}\left(\varepsilon(u)^{T} \varepsilon(v)\right) \quad \text { with } \quad \varepsilon(u)=\frac{1}{2}\left(\nabla u+\nabla u^{T}\right) .
$$

The functions $f: \Omega \rightarrow \mathbb{R}^{3}$ and $g: \partial \Omega_{N} \rightarrow \mathbb{R}^{3}$ denote volume and surface force, respectively. Let us note that our theory also holds for the case of almost incompressible linear elasticity; see [28] and the numerical results therein.

The domain $\Omega$ will be decomposed into $N$ nonoverlapping, open subdomains $\Omega_{i}$, $i=1, \ldots, N$. The interface $\Gamma$ is defined as the union of the subdomain boundaries without taking $\partial \Omega$ into account, i.e., $\Gamma:=\left\{x \in \bar{\Omega}_{i} \cap \bar{\Omega}_{j} ; i \neq j\right\}$. Then, the subdomains are each triangulated and then discretized by the finite element method with matching nodes on the interface. For simplicity, we use piecewise linear conforming finite elements.

In three dimensions, the interface can be decomposed into vertices, edges, and faces; for a detailed definition of these sets, cf. [40]. Edges and faces are defined as open sets. In a regular decomposition, vertices are the endpoints of edges. In general, this also applies to irregular decompositions. However, in these cases, edges with less than two vertices or vertices not being the endpoint of an edge can appear. A face between two arbitrary subdomains $\Omega_{i}$ and $\Omega_{j}$ will be denoted by $\mathcal{F}^{i j}$, while we denote edges between $\Omega_{i}, \Omega_{j}, \Omega_{l}$ and possibly more subdomains by $\mathcal{E}^{i l}$. Vertices of $\Omega_{i}$ that belong to multiple subdomains are denoted by $\mathcal{V}^{i k}$. 
Finally, we introduce the finite element spaces used for the FETI-DP and BDDC methods. For $i=1, \ldots, N$, by $W^{h}\left(\Omega_{i}\right)$ we denote the local finite element space on $\Omega_{i}$. The local trace space $W_{i}:=W^{h}\left(\Gamma_{i}\right)$ is defined on $\Gamma_{i}:=\overline{\Omega_{i}} \cap \Gamma$. We also introduce the global product space $W:=\Pi_{i=1}^{N} W_{i}$ and denote the space of functions that are continuous on the interface by $\widehat{W} \subset W$.

\section{FETI-DP and BDDC methods with a transformation of basis.}

3.1. Standard FETI-DP and BDDC. The FETI-DP method was originally introduced in $[17,18]$. To obtain the BDDC method, different authors proposed similar ways; see $[11,13,19]$.

We partition the degrees of freedom on the subdomains $\Omega_{i} \subset \Omega, i=1, \ldots, N$, into interior, dual, and primal degrees of freedom, denoted by $I, \Delta^{\prime}$, and $\Pi^{\prime}$, respectively. For subdomains $\Omega_{i}$ that share a part of the global boundary, i.e., $\partial \Omega_{i} \cap \partial \Omega \neq \emptyset$, their degrees of freedom on $\partial \Omega$ are assigned to the interior index set $I$. The assembled local stiffness matrices and the local load vectors of the subdomains $\Omega_{i}, i=1, \ldots, N$, then can be partitioned correspondingly. We need two different kinds of groupings of the previously introduced index sets $I, \Delta^{\prime}$, and $\Pi^{\prime}$, i.e., on the one hand, we group interior and dual variables denoted by the index $(\cdot)_{B^{\prime}}$, and, on the other hand, we group dual and primal indices denoted by the index $(\cdot)_{\Gamma}$. Then, there are multiple partitionings of the local stiffness matrices,

$$
K^{(i)}=:\left[\begin{array}{ccc}
K_{I I}^{(i)} & K_{\Delta^{\prime} I}^{(i) T} & K_{\Pi^{\prime} I}^{(i) T} \\
K_{\Delta^{\prime} I}^{(i)} & K_{\Delta^{\prime} \Delta^{\prime}}^{(i)} & K_{\Pi^{\prime} \Delta^{\prime}}^{(i) T} \\
K_{\Pi^{\prime} I}^{(i)} & K_{\Pi^{\prime} \Delta^{\prime}}^{(i)} & K_{\Pi^{\prime} \Pi^{\prime}}^{(i)}
\end{array}\right]\left\{\begin{array}{c}
=:\left[\begin{array}{ll}
K_{B^{\prime} B^{\prime}}^{(i)} & K_{\Pi^{\prime} B^{\prime}}^{(i) T} \\
K_{\Pi^{\prime} B^{\prime}}^{(i)} & K_{\Pi^{\prime} \Pi^{\prime}}^{(i)}
\end{array}\right], \\
=:\left[\begin{array}{cc}
K_{I I}^{(i)} & K_{I \Gamma}^{(i) T} \\
K_{I \Gamma}^{(i)} & K_{\Gamma \Gamma}^{(i)}
\end{array}\right]
\end{array}\right.
$$

In the same manner, the load vectors $f^{(i)}$ and the displacements $u^{(i)}, i=1, \ldots, N$, can be partitioned. We then introduce the block diagonal matrices

$$
K_{I I}:=\operatorname{diag}_{i=1}^{N} K_{I I}^{(i)}, \quad K_{B^{\prime} B^{\prime}}:=\operatorname{diag}_{i=1}^{N} K_{B^{\prime} B^{\prime}}^{(i)}, \quad K_{\Gamma \Gamma}:=\operatorname{diag}_{i=1}^{N} K_{\Gamma \Gamma}^{(i)}
$$

as well as the corresponding offdiagonal block $K_{\Gamma I}$. Again, the global right-hand side $f$ and the global displacement vector $u$ can be partitioned accordingly.

The inter-subdomain assembly operator $R_{\Pi^{\prime}}^{T}:=\left[R_{\Pi^{\prime}}^{(1) T}, \ldots, R_{\Pi^{\prime}}^{(N) T}\right]$, which performs the partial finite element assembly in the primal variables $u_{\Pi^{\prime}}^{(i)}$, is central to FETI-DP and BDDC. For BDDC, we also need $R_{\Delta^{\prime}}^{T}:=\left[R_{\Delta^{\prime}}^{(1) T}, \ldots, R_{\Delta^{\prime}}^{(N) T}\right]$, which performs the finite element assembly in the dual variables $u_{\Delta^{\prime}}^{(i)}$. For FETI-DP, instead of $R_{\Delta^{\prime}}^{T}$, we need a signed Boolean jump operator $B$ and its restriction to the interface $B_{\Gamma}=\left[B_{\Gamma}^{(1)}, \ldots, B_{\Gamma}^{(N)}\right]$, which has one +1 and one -1 per row such that $B_{\Gamma} u_{\Gamma}=0$ if and only if $u_{\Gamma}$ is continuous on the interface $\Gamma$.

Then, the FETI-DP master system is given by

$$
\left[\begin{array}{ccc}
K_{B^{\prime} B^{\prime}} & \widetilde{K}_{\Pi^{\prime} B^{\prime}}^{T} & B_{B^{\prime}}^{T} \\
\widetilde{K}_{\Pi^{\prime} B^{\prime}} & \widetilde{K}_{\Pi^{\prime} \Pi^{\prime}} & 0 \\
B_{B^{\prime}} & 0 & 0
\end{array}\right]\left[\begin{array}{c}
u_{B^{\prime}} \\
\widetilde{u}_{\Pi^{\prime}} \\
\lambda
\end{array}\right]=\left[\begin{array}{c}
\tilde{f} \\
0 \\
0
\end{array}\right],
$$

where we performed the partial assembly in the primal variables to couple the subdomain problems, i.e.,

$$
\widetilde{K}_{\Pi^{\prime} \Pi^{\prime}}=\sum_{i=1}^{N} R_{\Pi^{\prime}}^{(i) T} K_{\Pi^{\prime} \Pi^{\prime}}^{(i)} R_{\Pi^{\prime}}^{(i)}
$$




$$
\begin{aligned}
\widetilde{K}_{\Pi^{\prime} B^{\prime}} & =\left[R_{\Pi^{\prime}}^{(1) T} K_{\Pi^{\prime} B^{\prime}}^{(1)}, \ldots, R_{\Pi^{\prime}}^{(N) T} K_{\Pi^{\prime} B^{\prime}}^{(N)}\right], \\
\widetilde{f} & =\left[f_{B^{\prime}}^{T},\left(\sum_{i=1}^{N} R_{\Pi^{\prime}}^{(i) T} f_{\Pi^{\prime}}^{(i)}\right)^{T}\right]^{T},
\end{aligned}
$$

and

$$
B_{B^{\prime}}=\left[B_{B^{\prime}}^{(1)}, \ldots, B_{B^{\prime}}^{(N)}\right]
$$

the part of $B$ where the columns corresponding to primal variables were removed.

By proper block Gaussian elimination, we obtain the (unpreconditioned) standard FETIDP system $F \lambda=d$, where

$$
\begin{aligned}
F \lambda & :=\left(B_{B^{\prime}} K_{B^{\prime} B^{\prime}}^{-1} B_{B^{\prime}}^{T}+B_{B^{\prime}} K_{B^{\prime} B^{\prime}}^{-1} \widetilde{K}_{\Pi^{\prime} B^{\prime}}^{T} \widetilde{S}_{\Pi^{\prime} \Pi^{\prime}}^{-1} \widetilde{K}_{\Pi^{\prime} B^{\prime}} K_{B^{\prime} B^{\prime}}^{-1} B_{B^{\prime}}^{T}\right) \lambda \\
& =\left(B_{\Gamma} \widetilde{S}^{-1} B_{\Gamma}^{T}\right) \lambda \\
d: & =B_{B^{\prime}} K_{B^{\prime} B^{\prime}}^{-1} f_{B^{\prime}}+B_{B^{\prime}} K_{B^{\prime} B^{\prime}}^{-1} \widetilde{K}_{\Pi^{\prime} B^{\prime}}^{T} \widetilde{S}_{\Pi^{\prime} \Pi^{\prime}}^{-1}\left(\left(\sum_{i=1}^{N} R_{\Pi^{\prime}}^{(i) T} f_{\Pi^{\prime}}^{(i)}\right)-\widetilde{K}_{\Pi^{\prime} B^{\prime}} K_{B^{\prime} B^{\prime}}^{-1} f_{B^{\prime}}\right) .
\end{aligned}
$$

The Schur complement $\widetilde{S}_{\Pi^{\prime} \Pi^{\prime}}:=\widetilde{K}_{\Pi^{\prime} \Pi^{\prime}}-\widetilde{K}_{\Pi^{\prime} B^{\prime}}\left(K_{B^{\prime} B^{\prime}}\right)^{-1} \widetilde{K}_{\Pi^{\prime} B^{\prime}}^{T}$ represents the initial (a priori) coarse space. The Schur complement $\widetilde{S}$ is obtained from the local contributions $S^{(i)}:=\operatorname{diag}_{i=1}^{N}\left(K_{\Gamma \Gamma}^{(i)}-K_{\Gamma I}^{(i)}\left(K_{I I}^{(i)}\right)^{-1} K_{\Gamma I}^{(i) T}\right)$ assembled in the primal variables $\Pi^{\prime}$.

To define the BDDC method, it is convenient to introduce a modified ordering, i.e., $\left[u_{\Delta^{\prime}}^{(1)}, \ldots, u_{\Delta^{\prime}}^{(N)}, u_{\Pi^{\prime}}^{(1)}, \ldots, u_{\Pi^{\prime}}^{(N)}\right]$ instead of $\left[u_{\Delta^{\prime}}^{(1)}, u_{\Pi^{\prime}}^{(1)}, \ldots, u_{\Delta^{\prime}}^{(N)}, u_{\Pi^{\prime}}^{(N)}\right]$. We use the block diagonal matrix $\mathcal{K}_{\Gamma \Gamma}$ and $\mathcal{K}_{\Gamma I}$ to define the Schur complement on the interface $\Gamma$,

$$
\mathcal{S}_{\Gamma \Gamma}:=\mathcal{K}_{\Gamma \Gamma}-\mathcal{K}_{\Gamma I} K_{I I}^{-1} \mathcal{K}_{\Gamma I}^{T} .
$$

The right-hand side is obtained by the corresponding elimination of the interior degrees of freedom from $f$ and is denoted by $\left[g_{\Delta^{\prime}}^{T}, g_{\Pi^{\prime}}^{T}\right]^{T}$. The (unpreconditioned) BDDC system for $u_{\Gamma}^{T}=\left[u_{\Delta^{\prime}}^{T}, u_{\Pi^{\prime}}^{T}\right]^{T}$ with right-hand side $g^{T}=\left[g_{\Delta^{\prime}}^{T} R_{\Delta^{\prime}}, g_{\Pi^{\prime}}^{T} R_{\Pi^{\prime}}\right]^{T}$ writes

$$
\mathcal{S} u_{\Gamma}:=\left[\begin{array}{cc}
R_{\Delta^{\prime}}^{T} & 0 \\
0 & I_{\Pi^{\prime}}
\end{array}\right]\left[\begin{array}{cc}
I_{\Delta^{\prime}} & 0 \\
0 & R_{\Pi^{\prime}}^{T}
\end{array}\right] \mathcal{S}_{\Gamma \Gamma}\left[\begin{array}{cc}
I_{\Delta^{\prime}} & 0 \\
0 & R_{\Pi^{\prime}}
\end{array}\right]\left[\begin{array}{cc}
R_{\Delta^{\prime}} & 0 \\
0 & I_{\Pi^{\prime}}
\end{array}\right] u_{\Gamma}=g
$$

For heterogeneous problems, the use of an appropriate scaling is important. To be effective, this scaling has to depend on the coefficient. The scaling is of equal importance in adaptive FETI-DP and BDDC methods since, for a bad scaling, the resulting automatic coarse space can be very large [33].

In this paper, we will consider four different scalings. First, we introduce the standard $\rho$-scaling; see, e.g., [37, 39, 54, 57, 62]. For $x \in \Gamma_{i}$, we define $\mathcal{N}_{x}:=\left\{i \mid x \in \partial \Omega_{i}\right\}$. The coefficient evaluation is given by $\widehat{\rho}_{i}(x):=\sup _{x \in \operatorname{supp}\left(\varphi_{x}\right) \cap \Omega_{i}} \rho(x)$, where $\varphi_{x}$ is the nodal finite element function at $x$ and $\operatorname{supp}\left(\varphi_{x}\right)$ its support. Let $\Omega_{j}$ and $\Omega_{i}$ share either a face or an edge, and let $x \in \partial \Omega_{i} \cap \partial \Omega_{j}$. The corresponding nontrivial row of $B^{(j)}$ is then multiplied by the scaling $\delta_{i}^{\dagger}(x):=\widehat{\rho}_{i}(x) /\left(\sum_{k \in \mathcal{N}_{x}} \widehat{\rho}_{k}(x)\right)$ and vice versa. By this, we obtain the local scaling $D^{(j)}$. In BDDC the degrees of freedom on $\partial \Omega_{i}$ are scaled by $\delta_{i}^{\dagger}(x)$. This defines the BDDC scaling $D_{u}^{(i)}$. 
Second, we introduce the stiffness- (or K-, or super-lumped-) scaling [55], which is a heuristic approximation of the $\rho$-scaling. There, we replace the coefficient $\widehat{\rho}_{i}(x)$ by the corresponding diagonal element of the local stiffness matrix $K^{(i)}$.

Third, we introduce multiplicity-scaling [17,55], where the inverse of the multiplicity is used as a scaling. We obtain multiplicity-scaling from $\rho$-scaling by setting the coefficient to one.

Last, we consider deluxe-scaling, which was introduced recently; see, e.g, [4, 8, 10, 12, 14]. Deluxe-scaling is computationally expensive. Let us define the Schur complement of the stiffness matrix on the interface $\Gamma$, i.e.,

$$
S_{\Gamma \Gamma}:=\operatorname{diag}_{i=1}^{N} S^{(i)} .
$$

Then, consider either a face $\mathcal{F}^{i j}$ shared by the two subdomains $\Omega_{i}$ and $\Omega_{j}$ or an edge $\mathcal{E}^{i k}$ shared by the subdomains $\Omega_{i}, \Omega_{j}, \Omega_{k}$. Multiplicities greater than three can be handled analogously. Define for $l \in\{i, j\}$ the matrix $S_{\mathcal{F}^{i j}, 0}^{(l)}$ as the restriction of $S^{(l)}$ to the (open) face $\mathcal{F}^{i j}$. For a face $\mathcal{F}^{i j}$, in deluxe-scaling the nontrivial rows of $B^{(j)}$ corresponding to the Lagrange multipliers on this face are multiplied by $D_{u, \mathcal{F}^{i j}}^{(i) T}=\left(\left(S_{\mathcal{F}^{i j}, 0}^{(i)}+S_{\mathcal{F}^{i j}, 0}^{(j)}\right)^{-1} S_{\mathcal{F}^{i j}, 0}^{(i)}\right)^{T}$ if the orientation of the constraints in $B$ is chosen consistently. Otherwise, certain entries of $D_{u, \mathcal{F}^{i j}}^{(i)}$ have to be scaled by -1 . For $l \in\{i, j, k\}$, define $S_{\mathcal{E}^{i k}, 0}^{(l)}$ as the restriction of $S^{(l)}$ to the (open) edge $\mathcal{E}^{i k}$. For an edge $\mathcal{E}^{i k}$, in deluxe-scaling the nontrivial rows of $B^{(j)}$ corresponding to the Lagrange multipliers coupling $\Omega_{i}$ and $\Omega_{j}$ on this edge are multiplied by $D_{u, \mathcal{E}^{i k}}^{(i) T}=\left(\left(S_{\mathcal{E}^{i k}, 0}^{(i)}+S_{\mathcal{E}^{i k}, 0}^{(j)}+S_{\mathcal{E}^{i k}, 0}^{(k)}\right)^{-1} S_{\mathcal{E}^{i k}, 0}^{(i)}\right)^{T}$. Again, a consistent orientation of the Lagrange multipliers is assumed.

Choosing either of the scalings we can define the standard FETI-DP Dirichlet preconditioner (see [62])

$$
M_{D}^{-1}:=B_{D, \Gamma} R_{\Gamma}^{T} S_{\Gamma \Gamma} R_{\Gamma} B_{D, \Gamma}^{T}=B_{D, \Gamma} \widetilde{S} B_{D, \Gamma}^{T} .
$$

The standard BDDC preconditioner can be written

$$
M_{\mathrm{BDDC}}^{-1}:=\left[\begin{array}{cc}
R_{\Delta^{\prime}, D_{u}}^{T} R_{\Gamma} & 0 \\
0 & I_{\Pi^{\prime}}
\end{array}\right]\left[\begin{array}{cc}
K_{B^{\prime} B^{\prime}} & \widetilde{K}_{\Pi^{\prime} B^{\prime}}^{T} \\
\widetilde{K}_{\Pi^{\prime} B^{\prime}} & \widetilde{K}_{\Pi^{\prime} \Pi^{\prime}}
\end{array}\right]^{-1}\left[\begin{array}{cc}
R_{\Gamma}^{T} R_{\Delta^{\prime}, D_{u}} & 0 \\
0 & I_{\Pi^{\prime}}
\end{array}\right],
$$

where $B_{D, \Gamma}$ and $R_{\Delta^{\prime}, D_{u}}$ are the scaled variants $B_{\Gamma}$ and $R_{\Delta^{\prime}}$.

3.2. A standard transformation of basis for FETI-DP and BDDC. We briefly recall the standard transformation of basis (see, e.g., [35, 38, 40, 41, 45]), which, in the adaptive context, will be replaced by the generalized transformation of basis as described in [30].

Consider an edge $\mathcal{E}$ shared by $\Omega_{i}, \Omega_{j}$, and some other subdomains. It is intended to enforce the Krylov iterates $u$ to fulfill a constraint of the form

$$
c^{T}\left(u_{\mathcal{E}}^{(i)}-u_{\mathcal{E}}^{(j)}\right)=0 \quad \Leftrightarrow \quad c^{T} u_{\mathcal{E}}^{(i)}=c^{T} u_{\mathcal{E}}^{(j)}
$$

for a normalized constraint vector $c$. For $c$ different from any Euclidean basis vector, this corresponds to the use of a non-nodal basis function in the transformation of basis.

We then define a (square) transformation matrix $T_{\mathcal{E}}^{(l)}=\left[c, C^{(l) \perp}\right], l \in\{i, j\}$, where $C^{(l) \perp}$ is constructed such that the transformation is orthogonal. We then define the transformation matrix $T^{(l)}$ by extending $T_{\mathcal{E}}^{(l)}$ by the identity matrix outside of the edge. The transformed 
variables $\bar{u}^{(l)}$, stiffness matrices $\bar{K}^{(l)}$, and load vectors $\bar{f}^{(l)}$ on $\Omega_{l}$ then are

$$
\bar{K}^{(l)}=T^{(l) T} K^{(l)} T^{(l)}, \quad \bar{u}^{(l)}=T^{(l) T} u^{(l)}, \quad \bar{f}^{(l)}=T^{(l) T} f^{(l)}, \quad l \in\{i, j\} .
$$

After the basis transformation, the constraint is explicitly enforced by a partial subassembly in the new primal variables.

Applying the constraint vector to the displacements on the edge yields

$$
c^{T} u_{\mathcal{E}}^{(l)}=c^{T} T_{\mathcal{E}}^{(l)} \bar{u}_{\mathcal{E}}^{(l)}=c^{T}\left[c, C^{(l) \perp}\right] \bar{u}_{\mathcal{E}}^{(l)}=\bar{u}_{\mathcal{E}, 1}^{(l)}, \quad l \in\{i, j\},
$$

where $\bar{u}_{\mathcal{E}, 1}^{(l)}$ is the displacement at the first degree of freedom on the edge $\mathcal{E}$. A finite element assembly in this degree of freedom gives continuous values

$$
\widehat{u}_{\mathcal{E}, 1}:=\widehat{u}_{\mathcal{E}, 1}^{(i)}:=\widehat{u}_{\mathcal{E}, 1}^{(j)}:=\frac{1}{2} \bar{u}_{\mathcal{E}, 1}^{(i)}+\frac{1}{2} \bar{u}_{\mathcal{E}, 1}^{(j)}
$$

and (possibly) noncontinuous values $\widehat{u}_{\mathcal{E}, k}^{(l)}=\bar{u}_{\mathcal{E}, k}^{(l)}$ for $k>1, l \in\{i, j\}$. For the vectors that are transformed back to the original basis, we see that the constraint is enforced:

$$
c^{T}\left(T_{\mathcal{E}}^{(i)} \widehat{u}_{\mathcal{E}}^{(i)}-T_{\mathcal{E}}^{(j)} \widehat{u}_{\mathcal{E}}^{(j)}\right)=\frac{1}{2} \bar{u}_{\mathcal{E}, 1}^{(i)}+\frac{1}{2} \bar{u}_{\mathcal{E}, 1}^{(j)}-\left(\frac{1}{2} \bar{u}_{\mathcal{E}, 1}^{(i)}+\frac{1}{2} \bar{u}_{\mathcal{E}, 1}^{(j)}\right)=0 .
$$

This degree of freedom then belongs to the new set of primal variables $\Pi$.

3.3. An alternative formulation of the transformation of basis for FETI-DP and BDDC. In [40], a technique was introduced to avoid affecting the sparsity of the matrix $\bar{K}_{B^{\prime} B^{\prime}}^{(i)}$ by the explicit transformation of basis. This is important for face constraints. To resolve this issue, as in [40, Section 4.2.2], we can alternatively introduce additional, local Lagrange multipliers $\mu^{(i)}$ and consider local saddle point problems.

Let us briefly consider this in detail. By applying the operator $F$, the expression $B_{B^{\prime}} \bar{K}_{B^{\prime} B^{\prime}}^{-1} \bar{v}_{B^{\prime}}\left(\right.$ or $\left.B_{B^{\prime}}^{(i)}\left(\bar{K}_{B^{\prime} B^{\prime}}^{(i)}\right)^{-1} \bar{v}_{B^{\prime}}^{(i)}\right)$ has to be evaluated locally. Obviously, the minimization of $\bar{u}_{B^{\prime}}^{(i) T} \bar{K}_{B^{\prime} B^{\prime}}^{(i)} \bar{u}_{B^{\prime}}^{(i)}$ leads to the same result as the minimization of

$$
\left[\begin{array}{ll}
\bar{u}_{B^{\prime}}^{(i) T} & 0
\end{array}\right]\left[\begin{array}{ll}
\bar{K}_{B^{\prime} B^{\prime}}^{(i)} & \bar{K}_{\Pi^{\prime} B^{\prime}}^{(i) T} \\
\bar{K}_{\Pi^{\prime} B^{\prime}}^{(i)} & \bar{K}_{\Pi^{\prime} \Pi^{\prime}}^{(i)}
\end{array}\right]\left[\begin{array}{c}
\bar{u}_{B^{\prime}}^{(i)} \\
0
\end{array}\right]
$$

where the values at the additional primal variables $\Pi$ (cf. the previous Section 3.2) are set to zero. This is admissible since the jump operator is applied afterwards, and thus the values at the primal variables are set to zero.

Then, instead of minimizing the expression (3.5) in the transformed variables $\bar{u}_{B^{\prime}}^{(i)}$, we introduce a corresponding constraint $Q^{(i) T} u^{(i)}=0$ for the nontransformed variables. This consequently leads to the following saddle point problem

$$
\left[\begin{array}{ccc}
K_{I I}^{(i)} & K_{I \Gamma}^{(i)} & 0 \\
K_{I \Gamma}^{(i) T} & K_{\Gamma \Gamma}^{(i)} & Q^{(i)} \\
0 & Q^{(i) T} & 0
\end{array}\right]\left[\begin{array}{c}
u_{I}^{(i)} \\
u_{\Gamma}^{(i)} \\
\mu^{(i)}
\end{array}\right]=\left[\begin{array}{c}
v_{I}^{(i)} \\
v_{\Gamma}^{(i)} \\
0
\end{array}\right]
$$

with additional local Lagrange multipliers $\mu^{(i)}$ (cf. [40, Section 4.2.2] for more details), and where the right-hand side $\left(v_{I}^{(i) T}, v_{\Gamma}^{(i) T}\right)$ corresponds to $\left(\bar{v}_{B^{\prime}}^{T}, 0\right)$ transformed back to the initial basis and restricted to the local subdomain. 
Similar techniques of using saddle point problems to enforce the primal constraints have been used by other authors: in [18] the Lagrange multipliers are global; in [46, 47] the saddle point problems are local, which is also the case in our approach. In our case, however, the coarse Schur complement operator is assembled from the local subdomain contributions rather than built from a triple matrix product.

4. Adaptive FETI-DP and BDDC methods with a generalized transformation-ofbasis approach. In this section, we revisit the adaptive FETI-DP method [28, Section 5] and construct a corresponding method without using deflation or balancing. For highly heterogeneous problems, the a priori coarse space might not be sufficient to ensure convergence, and therefore adaptive strategies such as [28, Section 5] are needed. We will explain how to define the new basis vectors (in the space of displacements) for the generalized transformationof-basis approach [30] and how they are related to the deflation vectors used in [28] (which, for FETI-DP, are defined in the Lagrange multiplier space).

It is well known that deflation vectors are typically chosen as (approximate) eigenvectors corresponding to extreme eigenvalues of the operator in order to reduce the (effective) condition number of the deflated operator $[38,50,51]$. In adaptive domain decomposition, the deflation vectors are obtained from (approximately) solving local eigenvalue problems; see, e.g., [28] and the many other references cited in the introduction.

4.1. Generalized eigenvalue problems. Let us consider either a face $\mathcal{F}^{i j}$ between the subdomains $\Omega_{i}$ and $\Omega_{j}$ or an edge $\mathcal{E}^{i l}$ between the subdomains $\Omega_{i}$ and $\Omega_{l}$, where $\Omega_{i}$ and $\Omega_{l}$ have no common face; cf. Figure 4.1 (left). As in [47] for faces and [34] for faces and edges,

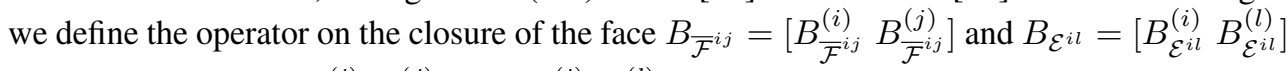
as the submatrix of $\left[B^{(i)} B^{(j)}\right]$ and $\left[B^{(i)} B^{(l)}\right]$, respectively, consisting of all the rows that contain exactly one +1 and one -1 . The operators $B_{\overline{\mathcal{F}}^{i j}}$ and $B_{\mathcal{E}^{i l}}$ provide the local jump on the closure of the face or edge, respectively.

Analogously, $B_{D, \overline{\mathcal{F}}^{i j}}=\left[B_{D, \overline{\mathcal{F}}^{i j}}^{(i)} B_{D, \overline{\mathcal{F}}^{i j}}^{(j)}\right]$ and $B_{D, \mathcal{E}^{i l}}=\left[B_{D, \mathcal{E}^{i l}}^{(i)} B_{D, \mathcal{E}^{i l}}^{(l)}\right]$ are the submatrices of $\left[B_{D}^{(i)} B_{D}^{(j)}\right]$ and $\left[B_{D}^{(i)} B_{D}^{(l)}\right]$, respectively. We also need

$$
P_{D, \overline{\mathcal{F}}^{i j}}:=B_{D, \overline{\mathcal{F}}^{i j}}^{T} B_{\overline{\mathcal{F}}^{i j}}, P_{D, \mathcal{E}^{i l}}:=B_{D, \mathcal{E}^{i l}}^{T} B_{\mathcal{E}^{i l}}, \quad \text { and } \quad S_{i s}:=\left[\begin{array}{cc}
S^{(i)} & 0 \\
0 & S^{(s)}
\end{array}\right], s \in\{j, l\} .
$$

We now establish and solve the following generalized eigenvalue problems that can be motivated by the localization of the global $P_{D}$ operator from the standard FETI-DP theory. We define the bilinear form $s_{i s}\left(u_{i s}, v_{i s}\right):=\left(S_{i s} u_{i s}, v_{i s}\right)$ with the displacement variables $u_{i s}, v_{i s} \in W_{i} \times W_{s}, s \in\{j, l\}$. The local generalized eigenvalue problem on either a face or an edge can then be formulated in variational form:

$$
\begin{aligned}
& \text { Find } w_{i s}^{k} \in\left(\operatorname{ker} S_{i s}\right)^{\perp} \text { with } \mu_{i s}^{k}>\text { TOL, such that } \\
& \text { (4.1) } \quad s_{i s}\left(P_{D, \overline{\mathcal{Z}}^{i s}} v_{i s}, P_{D, \overline{\mathcal{Z}}^{i s}} w_{i s}^{k}\right)=\mu_{i s}^{k} s_{i s}\left(v_{i s}, w_{i s}^{k}\right) \quad \forall v_{i s} \in\left(\operatorname{ker} S_{i s}\right)^{\perp}, s \in\{j, l\} ;
\end{aligned}
$$

cf. [47, Sections 3 and 4] and [28, Section 5] for a more detailed description.

We assume that we have now computed certain eigenvectors $w_{i s}^{k}$ for $\mu_{i s}^{k}>$ TOL, $s \in\{j, l\}$, on closed faces or edges. Later, during the FETI-DP iteration, we then enforce constraints of the form (see (4.1) and [28, Section 5])

$$
\left(w_{i s}, P_{D, \overline{\mathcal{Z}}^{i s}}^{T} S_{i s} P_{D, \overline{\mathcal{Z}}^{i s}} w_{i s}^{k}\right)=0 \quad \forall w_{i s} \in W_{i} \times W_{s}, s \in\{j, l\},
$$


by deflation or balancing (see [28] and [38]) or, as presented in the following, by a partial assembly after a transformation of basis on edges and faces.

Eigenvectors on closed faces. We define $u_{i j}^{k}=B_{D, \overline{\mathcal{F}}^{i j}} S_{i j} P_{D, \overline{\mathcal{F}}^{i j}} w_{i j}^{k}$ and decompose it into edge components $u_{i j, \mathcal{E}_{m}}^{k}$ and a component $u_{i j, \mathcal{F}}^{k}$ on the open face, all extended by zero to the closure (excluding the vertices) of the face; cf. [28, 29].

Eigenvectors on edges. The edge eigenvalue problems are crucial to obtain a convergence bound for heterogeneous problems in three dimensions [28]. For the theory in [28], edge eigenvalue problems are, however, only needed for edges which belong to more than three subdomains, i.e., with a multiplicity larger than three. If automatic mesh partitioners are used, then this applies to only a small percentage of edges. The methods proposed in [28], covered by a condition number bound, are therefore only slightly more expensive than the successful, classical face-based approaches from [47, 48], which only provided a condition number indicator.

4.2. Constraints for the generalized transformation of basis and solution spaces. For face eigenvalue problems, we obtain edge components and a component on the open face

$$
q_{i j, \mathcal{E}_{m}}^{k}:=B_{\overline{\mathcal{F}}^{i j}}^{T} u_{i j, \mathcal{E}_{m}}^{k} \quad \text { and } \quad q_{i j, \mathcal{F}}^{k}:=B_{\overline{\mathcal{F}}^{i j}}^{T} u_{i j, \mathcal{F}}^{k}
$$

and enforce the corresponding constraints on the displacement variable $w_{i j}$, i.e.,

$$
\begin{aligned}
\left(w_{i j}, q_{i j, \mathcal{E}_{m}}^{k}\right)=\left(w_{i j}, B_{\overline{\mathcal{F}}^{i j}}^{T} u_{i j, \mathcal{E}_{m}}^{k}\right) & =0, \quad m=1,2, \ldots, \\
\left(w_{i j}, q_{i j, \mathcal{F}}^{k}\right)=\left(w_{i j}, B_{\overline{\mathcal{F}}^{i j}}^{T} u_{i j, \mathcal{F}}^{k}\right) & =0 .
\end{aligned}
$$

Hence, we obtain the constraint vectors in the displacement space by a multiplication of the constraint vector in the Lagrange multiplier space by the localized version of the jump operator $B$, i.e., by $B_{\overline{\mathcal{F}}^{i j}}^{T}$.

For the edge eigenvalue problems, we choose constraint vectors

$$
q_{i l}^{k}:=B_{\mathcal{E}^{i l}}^{T} u_{i l}^{k}:=P_{D, \mathcal{E}^{i l}}^{T} S_{i l} P_{D, \mathcal{E}^{i l}} w_{i l}^{k}
$$

such that the constraint

$$
\left(w_{i l}, q_{i l}^{k}\right)=\left(w_{i l}, B_{\mathcal{E}^{i l}}^{T} u_{i l}^{k}\right)=0
$$

is satisfied. Thus, we obtain the constraint vectors in the displacement space by a multiplication of the constraint vector in the Lagrange multiplier space by the localized version of the jump operator $B$, i.e., by $B_{\mathcal{E}^{i l}}^{T}$.

We extend all constraint vectors $q_{i j, \mathcal{E}_{m}}^{k}, q_{i j, \mathcal{F}}^{k}$, and $q_{i l}^{k}$ by zero to $\widetilde{W}$. These vectors define the columns of the matrix $Q$. Analogously, we extend all constraint vectors $u_{i j, \mathcal{E}_{m}}^{k}, u_{i j, \mathcal{F}}^{k}$, and $u_{i l}^{k}$ by zero to the space of the Lagrange multipliers, defining the columns of the matrix $U$.

Obviously, the spaces

$$
\widetilde{W}_{Q}=\left\{w \in \widetilde{W}: Q^{T} w=0\right\} \quad \text { and } \quad \widetilde{W}_{U}=\left\{w \in \widetilde{W}: U^{T} B w=0\right\}
$$

are the same but they correspond to two different approaches to implement the constraints. The space $\widetilde{W}_{U}$ corresponds to the solution space for deflation or balancing; cf. [28]. In order to define the solution space for the generalized transformation of basis as introduced in [30], we have to introduce some notation and give some remarks. The solution space $\widetilde{W}_{\widehat{Q}}$ for the transformation-of-basis approach will result from a restriction of $\widetilde{W}_{Q}$. 
We now explain the necessary details that have to be considered when our adaptive constraints are implemented such that the restrictions of the generalized transformation-ofbasis approach [30] are satisfied. $q_{i l}^{k}$

Due to the form of the given constraints (see (4.3)-(4.5)), we have for $q_{i j, \mathcal{E}_{m}}^{k}, q_{i j, \mathcal{F}}^{k}$, and

$$
\begin{aligned}
\left(w_{i j}, q_{i j, \mathcal{E}_{m}}^{k}\right)=0 & \Leftrightarrow \quad\left(B_{\overline{\mathcal{F}}^{i j}}^{(i)} w^{(i)}, u_{i j, \mathcal{E}_{m}}^{k}\right)=-\left(B_{\overline{\mathcal{F}}^{i j}}^{(j)} w^{(j)}, u_{i j, \mathcal{E}_{m}}^{k}\right), \\
\left(w_{i j}, q_{i j, \mathcal{F}}^{k}\right)=0 & \Leftrightarrow \quad\left(B_{\overline{\mathcal{F}}^{i j}}^{(i)} w^{(i)}, u_{i j, \mathcal{F}}^{k}\right)=-\left(B_{\overline{\mathcal{F}}^{i j}}^{(j)} w^{(j)}, u_{i j, \mathcal{F}}^{k}\right), \\
\left(w_{i l}, q_{i l}^{k}\right)=0 & \Leftrightarrow \quad\left(B_{\mathcal{E}^{i l}}^{(i)} w^{(i)}, u_{i l}^{k}\right)=-\left(B_{\mathcal{E}^{i l}}^{(l)} w^{(l)}, u_{i l}^{k}\right) .
\end{aligned}
$$

Since $B_{\overline{\mathcal{F}}^{i j}}^{(i)}$ and $B_{\overline{\mathcal{F}}^{i j}}^{(j)}$ are closely related, i.e., both operators only differ by their sign when restricted to the face (i.e., $B_{\overline{\mathcal{F}}^{i j}}^{(i)}$ and $B_{\overline{\mathcal{F}}^{i j}}^{(j)}$ differ by their sign when all zero columns are removed), the constraint vector on the face will be identical for both sides of the face. The same arguments apply to the edge eigenvalue problems and the edge constraints from face eigenvalue problems since, again, $B_{\mathcal{E}^{i l}}^{(i)}$ and $-B_{\mathcal{E}^{i l}}^{(l)}$ are identical if restricted to the corresponding edge.

Given computed eigenvectors and resulting sets of orthonormalized constraints on a certain open face $\mathcal{F}^{i j}$ and on its related edges $\mathcal{E}_{m}$ or just on an edge $\mathcal{E}^{i l}$; cf. (4.3)-(4.5). The orthonormalized result will be denoted by $T_{\mathcal{F}^{i j}, \Pi}$ and $T_{\mathcal{E}_{m}, \Pi}$ or $T_{\mathcal{E}^{i l}, \Pi \text {. The matrices }}$ $T_{\mathcal{F}^{i j}}:=\left[T_{\mathcal{F}^{i j}, \Pi}, T_{\mathcal{F}^{i j}, \Delta}\right], T_{\mathcal{E}_{m}}=\left[T_{\mathcal{E}_{m}, \Pi}, T_{\mathcal{E}_{m}, \Delta}\right]$, and $T_{\mathcal{E}^{i l}}=\left[T_{\mathcal{E}^{i l}, \Pi}, T_{\mathcal{E}^{i l}, \Delta}\right]$ then are computed such that they are orthogonal matrices.

Given a face $\mathcal{F}^{i j}$, the sets of orthogonalized constraints $T_{\mathcal{F}^{i j}, \Pi}$ and $T_{\mathcal{E}_{m}, \Pi}$ can then be used as constraint vectors for both subdomains $\Omega_{i}$ and $\Omega_{j}$. This results from the form of $B$ and the fact that the constraint vector restricted to one subdomain equals $(-1)$ times the constraint vector restricted to the other subdomain; cf. (4.7). Given an edge $\mathcal{E}^{i l}\left(\mathcal{E}_{m}\right.$ can be handled likewise), the same applies for the two subdomains considered in the edge eigenvalue problem.

In order to satisfy the assumptions of the generalized transformation-of-basis approach, we also enforce the same constraints for all other jumps between two arbitrary subdomains at the considered face or edge. Then, for a face $\mathcal{F}^{i j}$ with jumps $w^{(i)}-w^{(j)}$ and edges $\mathcal{E}_{m}$ or an edge $\mathcal{E}^{i l}$ and all jumps $w^{(i)}-w^{(s)}$ across the edge, we have that the local transformations are equal for all subdomains sharing either the face or the edge, i.e.,

$$
T_{\mid \mathcal{F}^{i j}}^{(i)}=T_{\mid \mathcal{F}^{i j}}^{(j)}=T_{\mathcal{F}^{i j}}, \quad T_{\mid \mathcal{E}_{m}}^{(i)}=T_{\mid \mathcal{E}_{m}}^{(s)}=T_{\mathcal{E}_{m}}, \quad T_{\mid \mathcal{E}^{i l}}^{(i)}=T_{\mid \mathcal{E}^{i l}}^{(s)}=T_{\mathcal{E}^{i l}}
$$

and all pairs of subdomains $\left\{\Omega_{i}, \Omega_{s}\right\}$ sharing the edges $\mathcal{E}_{m}$ or $\mathcal{E}^{i l}$.

Thus, the constraint set obtained from our local eigenvalue problems can be extended such that the local transformations satisfy condition (4.8) for the generalized transformation of basis as given in [30]. We then have $\widehat{Q}:=[Q, *]$ with range $Q \subset$ range $\widehat{Q}$ and the solution space

$$
\widetilde{W}_{\widehat{Q}}=\left\{w \in \widetilde{W}: \widehat{Q}^{T} w=0\right\} \subset \widetilde{W}_{Q}=\widetilde{W}_{U} ;
$$

cf. the definitions in (4.6). The constraints are then enforced by a partial subassembly; cf. Figure 4.1. Therefore, the solution space $\widetilde{W}_{\widehat{Q}}$ is in general a strict subset of the solution space $\widetilde{W}_{U}$, and we obtain our $P_{D}$-estimate from [28, Lemma 6.1] for $\widetilde{W}_{\widehat{Q}}$, i.e., for $w \in \widetilde{W}_{\widehat{Q}}$, we have

$$
\left|P_{D} w\right|_{\widetilde{S}}^{2} \leq 4 \max \left\{N_{\mathcal{F}}, N_{\mathcal{E}} M_{\mathcal{E}}\right\}^{2} \mathrm{TOL}|w|_{\widetilde{S}}^{2}
$$



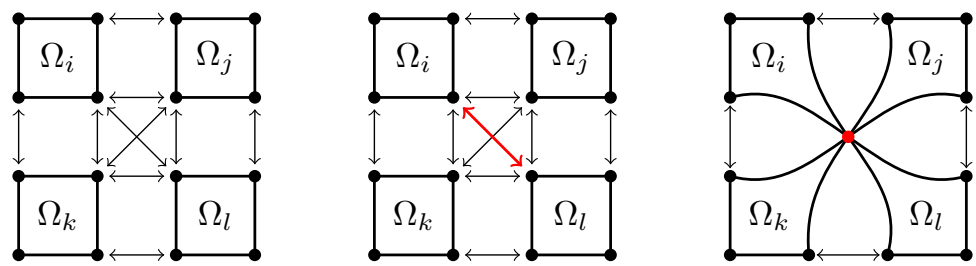

FIGURE 4.1. Cross sectional view of four subdomains sharing an edge. Arrows symbolize redundant Lagrange multipliers in FETI-DP (left). Assume that, using deflation, one primal constraint is introduced, involving the Lagrange multiplier depicted in bold red color (middle). Using partial assembly, after a generalized transformation of basis, the primal constraint is now enforced between all four subdomains, effectively removing all six Lagrange multipliers (right).

In our implementation, the constrained elements of the space $\widetilde{W}_{\widehat{Q}}$ are represented by $\widetilde{W}_{T, a}:=\left\{\widehat{w}=R_{\mu}^{T} T^{T} w: w \in \widetilde{W}\right\}$, where $R_{\mu}^{T}=\left(R^{T} R\right)^{-1} R^{T}, T=\operatorname{blockdiag}_{i=1, \ldots, N} T^{(i)}$. This requires the definition of $R^{T}$ and $T^{(i)}, i=1, \ldots, n$. The operator $R^{T}$ assembles in all a posteriori degrees of freedom and leaves all other degrees of freedom (interior, a priori primal and remaining dual variables) unchanged. The operator $T^{(i)}$ reduces to the identity on all interior degrees of freedom and is blockdiagonal on the interface with blocks $T_{\mathcal{F}^{i j}}$ and $T_{\mathcal{E}^{i l}}$ for all faces $\mathcal{F}^{i j}$ and edges $\mathcal{E}^{i l}$ shared by $\Omega_{i}$.

4.3. Adaptive FETI-DP and BDDC operators for the generalized transformation-ofbasis approach. In [30], FETI-DP and BDDC methods using a generalized transformationof-basis approach were introduced.

Using this approach and the notation from [30], the preconditioned adaptive FETI-DP method is then given by

$$
\begin{aligned}
& \widehat{M}_{T}^{-1} \widehat{F} \lambda:=\left(\widehat{B}_{D} \widehat{\widetilde{S}} \widehat{B}_{D}^{T}\right)\left(\widehat{B} \widehat{\widetilde{S}}^{-1} \widehat{B}^{T}\right) \lambda \\
& :=\left(B_{D} T R_{\mu}\left(R^{T} T^{T} \widetilde{S} T R\right) R_{\mu}^{T} T^{T} B_{D}^{T}\right)\left(B T R\left(R^{T} T^{T} \widetilde{S} T R\right)^{-1} R^{T} T^{T} B^{T}\right) \lambda=\widehat{d} .
\end{aligned}
$$

Introducing another operator $R^{\prime T}$ which assembles in all remaining dual and a posteriori primal variables and leaves the a priori primal variables unchanged, the preconditioned adaptive BDDC method is given by

$$
\begin{aligned}
\widehat{M}_{\mathrm{BDDC}}^{-1} \mathcal{S} u & :=\left(R^{\prime T} T \widehat{D}_{u} R \widehat{\widetilde{S}}^{-1} R^{T} \widehat{D}_{u} T^{T} R^{\prime}\right)\left(R^{\prime T} \widetilde{S} R^{\prime}\right) u \\
: & =\left(R^{\prime T} D_{u} T R\left(R^{T} T^{T} \widetilde{S} T R\right)^{-1} R^{T} T^{T} D_{u} R^{\prime}\right)\left(R^{\prime T} \widetilde{S} R^{\prime}\right) u=\widehat{g}
\end{aligned}
$$

with $D_{u}$ the degree of freedom scaling in BDDC corresponding to the Lagrange multiplier scaling $D$ in FETI-DP.

4.4. Condition number estimate for adaptive FETI-DP and BDDC. We can now formulate the following theorem for our adaptive algorithm using a generalized transformation of basis based on [30].

In [30], a correspondence of FETI-DP or BDDC methods using a generalized transformation of basis and of FETI-DP methods using deflation or balancing was shown. These methods have essentially the same eigenvalues, and as opposed to the previous theory, this result also applies to general scalings and heterogeneous problems including coefficient jumps inside subdomains.

THEOREM 4.1. Let $N_{\mathcal{F}}$ denote the maximum number of faces of a subdomain, $N_{\mathcal{E}}$ the maximum number of edges of a subdomain, $M_{\mathcal{E}}$ the maximum multiplicity of an edge, and TOL 

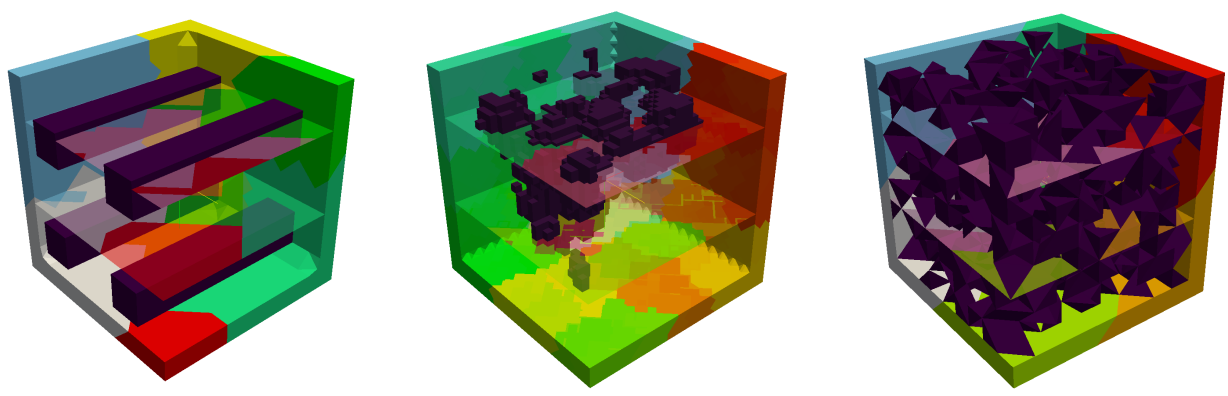

FIGURE 5.1. Irregular decomposition of the unit cube with composite material (left), representative volume element (center), and randomized coefficients (right). High coefficients $\mathrm{E}_{2}=1 \mathrm{e}+06$ (for composite and random) and $\mathrm{E}_{2}=2.1 \mathrm{e}+05$ (for the RVE) are shown in a dark purple color in the picture, subdomains shown in different colors in the background and by half-transparent slices. Visualization for $N=8$ and $1 / h=3 N^{1 / 3}$ (left), $N=512$ and $1 / h=4 N^{1 / 3}$ (center), and $N=8$ and $1 / h=5 N^{1 / 3}$ (right).

a given tolerance for solving the local generalized eigenvalue problems (4.1). Furthermore, let all vertices chosen to be primal. Then, the condition number $\kappa\left(\widehat{M}_{T}^{-1} \widehat{F}\right)$ of the FETI-DP algorithm with constraints enforced by the generalized transformation of basis as described satisfies

$$
\kappa\left(\widehat{M}_{T}^{-1} \widehat{F}\right) \leq 4 \max \left\{N_{\mathcal{F}}, N_{\mathcal{E}} M_{\mathcal{E}}\right\}^{2} \mathrm{TOL} .
$$

The condition number $\kappa\left(\widehat{M}_{B D D C}^{-1} \mathcal{S}\right)$ of the BDDC algorithm with adaptive constraints enforced by the generalized transformation of basis as described above satisfies

$$
\kappa\left(\widehat{M}_{B D D C}^{-1} \mathcal{S}\right) \leq 4 \max \left\{N_{\mathcal{F}}, N_{\mathcal{E}} M_{\mathcal{E}}\right\}^{2} \text { TOL }
$$

Proof. The proof is complete by acknowledging that this is a special case of [30, Theorem 6.7] and [30, Theorem 7.3] if the transformations are built according to [30] (i.e., they are identical for all sides of any considered edge) and by using (4.10).

Note that the bound in (4.1) is algebraic in the sense that the condition number bound holds under very weak assumptions. However, under unfavorable conditions, the coarse space can be so large that the method reduces to a direct solver.

REMARK 4.2. Note that the explicit condition number of the FETI-DP or BDDC method with our generalized transformation-of-basis approach is always smaller or equal to that of the corresponding balancing or deflation approach. This results from the fact that in our generalized transformation-of-basis approach in the partial assembly, we often enforce additional constraints compared to the deflation approach-without creating a larger coarse space; cf. (4.9), (4.8), and Figure 4.1.

Nevertheless, note that we can always find an equivalent balancing or deflation method by expanding the constraint columns $U$ such that all the constraints from $\widetilde{W}_{\widehat{Q}}$ are implemented and such that $\kappa\left(M_{P P}^{-1} F\right)=\kappa\left(\widehat{M}_{T}^{-1} \widehat{F}\right)$; cf. [30].

5. Numerical results. In this section, we provide numerical results for the adaptive coarse spaces presented in [28] (including the classical variants of $[47,48]$ relying on face eigenvalue problems only) but implemented by the generalized transformation-of-basis approach for FETI-DP and BDDC.

As in [28], we consider compressible linear elasticity on the unit cube $\Omega=[0,1]^{3}$ as a model problem. The Poisson ratio is $\nu=0.3$ and the Young Modulus is $\mathrm{E} \in\{1,1 \mathrm{e}+06\}$. We assume constant values of $\mathrm{E}$ and $\nu$ on each finite element. 


\section{ETNA}

Kent State University and

Johann Radon Institute (RICAM)
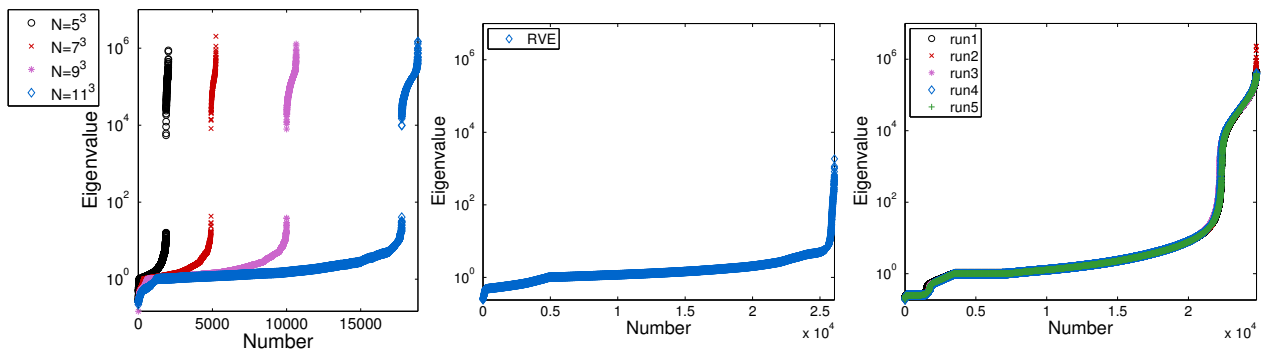

FIGURE 5.2. Local eigenvalues greater than 0.1 from all generalized eigenvalue problems (4.1) for some simulations for the three materials depicted in Figure 5.1: Composite material (left), representative volume element (center), and randomized coefficients for $N=216$ and the first five runs (right).

The initial coarse space for all methods consists of vertex constraints. We then add several other vertices such that each nonstraight edge has at least three primal vertices. This choice is identical to that of [28].

We consider irregular decompositions of the unit cube. These decompositions are obtained from the METIS graph partitioner (see [25]) using the options -ncommon=3 and -contig to avoid noncontiguous subdomains and unwanted hinge modes between sets of tetrahedra inside single subdomains.

We test five different algorithms for FETI-DP and BDDC; cf. [28]. The first three, Algorithms Ia, Ib, and Ic originate from our recent paper [28] but are using a transformation of basis instead of balancing. Algorithm Ia uses all the constraints obtained from the local eigenvalue problems. Algorithm Ib and Algorithm Ic are heuristically reduced variants of Algorithm Ia. For Algorithm Ib, edge eigenvalue problems are discarded if there are no jumps of the Young Modulus present within a distance of one finite element around the edges. In Algorithm Ic not only edge eigenvalue problems are discarded on these edges but also edge constraints from face eigenvalue problems. The Algorithms II and III are the classical face-based algorithms from [47, 48]. There, Algorithm II uses all constraints (edge and face constraints) obtained from face eigenvalue problems but still lacks any edge eigenvalue problem. Algorithm III was already tested in [48] and just uses face constraints from face eigenvalue problems.

In all tables, we denote by $\kappa$ the condition number of the preconditioned FETI-DP and BDDC operator, and its denotes the number of preconditioned conjugate gradient (pcg) iterations that are needed until convergence. The iteration is also stopped if no convergence is observed within 500 iterations. As a stopping criterion, we require the pcg algorithm to reduce the preconditioned starting residual by $10^{-10}$. The condition numbers $\kappa$ given in the table are the standard estimates obtained from pcg. The condition number estimates for FETI-DP and BDDC differ slightly since, in most cases, the estimates of the smallest eigenvalue differ slightly, typically starting in the second or third digit.

By $\left|\Pi^{\prime}\right|$ we denote the size of the standard coarse space while by $|\Pi|$ we indicate the size of the corresponding adaptive coarse space implemented by the transformation of basis; see the previous sections and [30]. By $N$ we denote the number of subdomains. For our coarse spaces introduced in [28], we also present $\# \mathcal{E}_{\text {evp }}$, the number of edge eigenvalue problems, and in parentheses the percentage of these in the total number of eigenvalue problems.

For all runs, we highlight small condition numbers, i.e., $\kappa \leq 50$, in bold face. If not stated otherwise, all eigenvalue problems are solved by the MATLAB builtin "eig" function; cf. Section 5.1.4 and Section 5.3 for a solution of the eigenvalue problems by the LOBPCG solver 
$[42,43]$. Except in Table 5.9, we always use $\mathrm{TOL}=10$. The resulting condition number is typically at the order of TOL; see also [28, p. A2893].

When $\rho$-scaling is used we apply the standard $\rho$-scaling, where the nodal coefficient values are given by the maximum value of the coefficient function on all neighboring tetrahedra inside the same subdomain.

We enforce zero Dirichlet boundary conditions for the face with $x=0$ and zero Neumann boundary conditions elsewhere and apply the body force $f=(0.1,0.1,0.1)^{T}$.

The following section has three subsections. In Section 5.1, we test the transformation-ofbasis approach for materials already tested with $\rho$-scaling and the balancing approach in [28]. In Section 5.2, we test our composite material and randomly distributed coefficients with different scalings such as $\rho$-, deluxe-, stiffness-/K-, and multiplicity-scaling. In Section 5.3, we present more results in order to demonstrate the robustness of our algorithm with the LOBPCG eigensolver [42, 43].

5.1. Comparison to the balancing method. In this section, we test three different distributions of the Young Modulus E; see Figure 5.1, the more detailed descriptions in the following Sections 5.1.1-5.1.3, or our recent paper [28].

5.1.1. Composite material. In this section, we consider a composite material. The material consists of a soft matrix material with $\mathrm{E}=1$ that surrounds $N^{2 / 3}$ many beams that run in a straight line from the face with $x=0$ to the face with $x=1$ and that occupy $1 / 9$ of that cross-section of the material; cf. Figure 5.1 and [28] where we referred to this material as composite material $\mathbf{1}$.

We see that FETI-DP and BDDC behave almost identically. Clearly, the coarse spaces are of the same size and the iteration count is almost the same for both methods. We see that the size of the second-level coarse space is quite modest compared to the standard coarse space. Compared to the coarse space implemented by the balancing approach (cf. [28, Table 8.2]), the coarse space by the transformation of basis in Table 5.1 is up to two times the size of the previous one. This is due to the fact that we always make all three degrees of freedom primal that belong to a node, even if only one or two constraints are required. On the other hand, the coarse spaces of [28, Table 8.3] and Table 5.2 do have a comparable size regardless of the implementation.

For a more detailed consideration of the computational overhead of additional edge eigenvalue problems, see the very detailed discussion in [28]. For a distribution of the local eigenvalues for some of the runs; see Figure 5.2 (left).

5.1.2. Representative volume element. In this section, we consider a representative volume element of a modern steel microstructure; see Figure 5.1. The RVE has been obtained from the one in [44, Fig. 5.5] by resampling; see [28, Section 8.2]. As in [44], we use $\nu=0.3$, $\mathrm{E}_{1}=210$, and $\mathrm{E}_{2}=210000$ as artificial material parameters. The FETI-DP and BDDC algorithms tested here and the FETI-DP algorithm with a balancing approach (see [28]) show almost identical convergence behavior. The transformation-of-basis approach gives a larger coarse space. As mentioned in the previous section this could be improved so that the balancing approach does not have significant advantages over the transformation approach; we did not do so because of the ease of implementation. For a distribution of the local eigenvalues, see Figure 5.2 (center).

5.1.3. Randomly distributed coefficients. In this section, we test randomly distributed coefficients. We let $20 \%$ of the tetrahedra in the unit cube take the value $\mathrm{E}=1 \mathrm{e}+06$ while the other tetrahedra take $\mathrm{E}=1$. Both, FETI-DP and BDDC behave almost identically. The coarse spaces of transformation and balancing are of comparable size; cf. [28] for $N \in\left\{4^{3}, 5^{3}\right\}$. Compared to [28], we now show results for $N=6^{3}$ where it can also be seen that not only 


\section{ETNA}

Kent State University and

Johann Radon Institute (RICAM)

TABLE 5.1

Compressible linear elasticity with $\mathrm{E}_{1}=1, \mathrm{E}_{2}=1 \mathrm{e}+06$. Coarse spaces for $T O L=10$ for all generalized eigenvalue problems.

\begin{tabular}{|c|c|c|c|c|c|c|c|c|c|c|c|c|}
\hline \multicolumn{13}{|c|}{ FETI-DP } \\
\hline & & \multicolumn{5}{|c|}{ Algorithm Ia, Ib, and Ic } & \multicolumn{3}{|c|}{ Algorithm II } & \multicolumn{3}{|c|}{ Algorithm III } \\
\hline$N$ & $\left|\Pi^{\prime}\right|$ & & $\kappa$ & its & $|\Pi|$ & $\# \mathcal{E}_{e v p}$ & $\kappa$ & its & $|\Pi|$ & $\kappa$ & its & $|\Pi|$ \\
\hline \multirow{3}{*}{$3^{3}$} & & a) & 8.44 & 29 & 144 & $7(11.9 \%)$ & \multirow{3}{*}{8.44} & \multirow{3}{*}{29} & \multirow{3}{*}{144} & \multirow{3}{*}{$8.31 \mathrm{e}+05$} & \multirow{3}{*}{51} & \multirow{3}{*}{90} \\
\hline & 615 & b) & 8.44 & 29 & 144 & $4(7.1 \%)$ & & & & & & \\
\hline & & c) & 8.44 & 29 & 129 & $4(7.1 \%)$ & & & & & & \\
\hline \multirow{3}{*}{$5^{3}$} & & a) & 14.30 & 36 & 459 & $14(5.2 \%)$ & \multirow{3}{*}{14.30} & \multirow{3}{*}{36} & \multirow{3}{*}{453} & \multirow{3}{*}{$3.29 \mathrm{e}+05$} & \multirow{3}{*}{187} & \multirow{3}{*}{303} \\
\hline & 3084 & b) & 14.30 & 36 & 459 & $8(3.0 \%)$ & & & & & & \\
\hline & & c) & 14.30 & 37 & 375 & $8(3.0 \%)$ & & & & & & \\
\hline \multirow{3}{*}{$7^{3}$} & & a) & 13.92 & 40 & 1098 & $48(6.0 \%)$ & \multirow{3}{*}{$2.93 e+05$} & \multirow{3}{*}{84} & \multirow{3}{*}{1074} & \multirow{3}{*}{$2.96 \mathrm{e}+05$} & \multirow{3}{*}{373} & \multirow{3}{*}{780} \\
\hline & 8781 & b) & 13.92 & 40 & 1089 & $21(2.7 \%)$ & & & & & & \\
\hline & & c) & 13.93 & 41 & 942 & $21(2.7 \%)$ & & & & & & \\
\hline \multirow{3}{*}{$9^{3}$} & & a) & 16.27 & 41 & 2070 & $90(5.2 \%)$ & \multirow{3}{*}{$2.66 \mathrm{e}+05$} & \multirow{3}{*}{71} & \multirow{3}{*}{2043} & \multirow{3}{*}{$4.69 e+05$} & \multirow{3}{*}{482} & \multirow{3}{*}{1572} \\
\hline & 19029 & b) & 16.28 & 42 & 2067 & $45(2.7 \%)$ & & & & & & \\
\hline & & c) & 16.28 & 42 & 1812 & $45(2.7 \%)$ & & & & & & \\
\hline & & a) & 15.05 & 43 & 3582 & $167(5.2 \%)$ & & & & & & \\
\hline $11^{3}$ & 35214 & b) & 15.05 & 43 & 3570 & $95(3.0 \%)$ & $2.66 \mathrm{e}+05$ & 142 & 3504 & $3.60 \mathrm{e}+05$ & 500 & 2724 \\
\hline & & c) & 15.05 & 43 & 3192 & $95(3.0 \%)$ & & & & & & \\
\hline & & a) & 17.12 & 44 & 5895 & $303(5.6 \%)$ & & & & & & \\
\hline $13^{3}$ & 58179 & b) & 17.12 & 44 & 5889 & $171(3.3 \%)$ & $2.74 \mathrm{e}+05$ & 225 & 5739 & $3.01 \mathrm{e}+05$ & 500 & 4557 \\
\hline & & c) & 17.13 & 44 & 5346 & $171(3.3 \%)$ & & & & & & \\
\hline
\end{tabular}

\begin{tabular}{|c|c|c|c|c|c|c|c|c|c|c|c|c|}
\hline \multicolumn{13}{|c|}{ BDDC } \\
\hline & & \multicolumn{5}{|c|}{ Algorithm Ia, Ib, and Ic } & \multicolumn{3}{|c|}{ Algorithm II } & \multicolumn{3}{|c|}{ Algorithm III } \\
\hline$N$ & $\left|\Pi^{\prime}\right|$ & & $\kappa$ & its & $|\Pi|$ & $\# \mathcal{E}_{e v p}$ & $\kappa$ & its & $|\Pi|$ & $\kappa$ & its & $|\Pi|$ \\
\hline \multirow{3}{*}{$3^{3}$} & & a) & 8.52 & 29 & 144 & $7(11.9 \%)$ & \multirow{3}{*}{8.52} & \multirow{3}{*}{29} & \multirow{3}{*}{144} & \multirow{3}{*}{$8.42 \mathrm{e}+05$} & \multirow{3}{*}{66} & \multirow{3}{*}{90} \\
\hline & 615 & b) & 8.52 & 29 & 144 & $4(7.1 \%)$ & & & & & & \\
\hline & & c) & 8.52 & 29 & 129 & $4(7.1 \%)$ & & & & & & \\
\hline \multirow{3}{*}{$5^{3}$} & & a) & 14.44 & 35 & 459 & $14(5.2 \%)$ & \multirow{3}{*}{14.44} & \multirow{3}{*}{37} & \multirow{3}{*}{453} & \multirow{3}{*}{$3.33 \mathrm{e}+05$} & \multirow{3}{*}{241} & \multirow{3}{*}{303} \\
\hline & 3084 & b) & 14.44 & 35 & 459 & $8(3.0 \%)$ & & & & & & \\
\hline & & c) & 14.45 & 36 & 375 & $8(3.0 \%)$ & & & & & & \\
\hline \multirow{3}{*}{$7^{3}$} & & a) & 14.08 & 40 & 1098 & $48(6.0 \%)$ & \multirow{3}{*}{$2.97 \mathrm{e}+05$} & \multirow{3}{*}{98} & \multirow{3}{*}{1074} & \multirow{3}{*}{$3.00 \mathrm{e}+05$} & \multirow{3}{*}{459} & \multirow{3}{*}{780} \\
\hline & 8781 & b) & 14.08 & 40 & 1089 & $21(2.7 \%)$ & & & & & & \\
\hline & & c) & 14.08 & 41 & 942 & $21(2.7 \%)$ & & & & & & \\
\hline \multirow{3}{*}{$9^{3}$} & & a) & 16.44 & 41 & 2070 & $90(5.2 \%)$ & \multirow{3}{*}{$2.69 \mathrm{e}+05$} & \multirow{3}{*}{76} & \multirow{3}{*}{2043} & \multirow{3}{*}{$4.75 e+05$} & \multirow{3}{*}{500} & \multirow{3}{*}{1572} \\
\hline & 19029 & b) & 16.44 & 41 & 2067 & $45(2.7 \%)$ & & & & & & \\
\hline & & c) & 16.44 & 42 & 1812 & $45(2.7 \%)$ & & & & & & \\
\hline & & a) & 15.22 & 40 & 3582 & $167(5.2 \%)$ & & & & & & \\
\hline $11^{3}$ & 35214 & b) & 15.22 & 41 & 3570 & $95(3.0 \%)$ & $2.69 \mathrm{e}+05$ & 162 & 3504 & $3.72 \mathrm{e}+05$ & 500 & 2724 \\
\hline & & c) & 15.22 & 42 & 3192 & $95(3.0 \%)$ & & & & & & \\
\hline & & a) & 17.32 & 41 & 5895 & $303(5.6 \%)$ & & & & & & \\
\hline $13^{3}$ & 58179 & b) & 17.32 & 41 & 5889 & $171(3.3 \%)$ & $2.77 \mathrm{e}+05$ & 250 & 5739 & $3.40 \mathrm{e}+05$ & 500 & 4557 \\
\hline & & c) & 17.32 & 41 & 5346 & $171(3.3 \%)$ & & & & & & \\
\hline
\end{tabular}

the condition number but also the iteration count of Algorithm II deteriorates with larger $N$. Therefore, we also include the maximum and minimum value in the table. For a distribution of the local eigenvalues for some of the runs, see Figure 5.2 (right).

5.1.4. Composite material using LOBPCG as an eigenvalue solver. In this section, we present results for the FETI-DP and BDDC algorithm in combination with the iterative LOBPCG (cf. [42, 43]) eigenvalue solver. We choose a block size of 10 and use a Cholesky decomposition of the right-hand side of the eigenvalue problem as local preconditioner. We limit the number of maximum iterations of the iterative solver as indicated in the table. We test a larger example than in the previous paper [28], i.e., $N=5^{3}$, to show that a larger admissible number of iterations does not consequently lead to faster convergence; see the results for 


\section{ETNA}

Kent State University and

Johann Radon Institute (RICAM)

TABLE 5.2

Compressible linear elasticity with $\mathrm{E}_{1}=1, \mathrm{E}_{2}=1 \mathrm{e}+06$. Coarse spaces for $T O L=10$ for all generalized eigenvalue problems.

\begin{tabular}{|c|c|c|c|c|c|c|c|c|c|c|c|c|}
\hline \multicolumn{13}{|c|}{ Composite material, irregular partitioning, } \\
\hline \multicolumn{13}{|c|}{$\begin{array}{ll}\text { FETI-DP } \\
\end{array}$} \\
\hline & & \multicolumn{5}{|c|}{ Algorithm Ia, Ib, and Ic } & \multicolumn{3}{|c|}{ Algorithm II } & \multicolumn{3}{|c|}{ Algorithm III } \\
\hline$N$ & $\left|\Pi^{\prime}\right|$ & & $\kappa$ & its & $|\Pi|$ & $\# \mathcal{E}_{e v p}$ & $\kappa$ & its & $|\Pi|$ & $\kappa$ & its & $|\Pi|$ \\
\hline \multirow{3}{*}{$3^{3}$} & & a) & 8.65 & 34 & 699 & $2(2.0 \%)$ & \multirow{3}{*}{8.65} & \multirow{3}{*}{34} & \multirow{3}{*}{699} & \multirow{3}{*}{$1.36 \mathrm{e}+06$} & \multirow{3}{*}{68} & \multirow{3}{*}{243} \\
\hline & 960 & b) & 8.65 & 34 & 699 & $1(1.0 \%)$ & & & & & & \\
\hline & & c) & 8.67 & 34 & 450 & $1(1.0 \%)$ & & & & & & \\
\hline \multirow{3}{*}{$5^{3}$} & & a) & 9.16 & 35 & 3675 & $25(4.2 \%)$ & \multirow{3}{*}{9.16} & \multirow{3}{*}{35} & \multirow{3}{*}{3669} & \multirow{3}{*}{$5.50 \mathrm{e}+05$} & \multirow{3}{*}{190} & \multirow{3}{*}{1242} \\
\hline & 5433 & b) & 9.16 & 35 & 3675 & $12(2.1 \%)$ & & & & & & \\
\hline & & c) & 10.49 & 36 & 2325 & $12(2.1 \%)$ & & & & & & \\
\hline \multirow{3}{*}{$7^{3}$} & & a) & 10.76 & 37 & 10101 & $65(3.6 \%)$ & \multirow{3}{*}{10.76} & \multirow{3}{*}{37} & \multirow{3}{*}{10089} & \multirow{3}{*}{$1.21 \mathrm{e}+06$} & \multirow{3}{*}{424} & \multirow{3}{*}{3606} \\
\hline & 16248 & b) & 10.76 & 37 & 10101 & $27(1.5 \%)$ & & & & & & \\
\hline & & c) & 13.36 & 39 & 6693 & $27(1.5 \%)$ & & & & & & \\
\hline
\end{tabular}

\begin{tabular}{|c|c|c|c|c|c|c|c|c|c|c|c|c|}
\hline \multicolumn{13}{|c|}{ BDDC } \\
\hline & & \multicolumn{5}{|c|}{ Algorithm Ia, Ib, and Ic } & \multicolumn{3}{|c|}{ Algorithm II } & \multicolumn{3}{|c|}{ Algorithm III } \\
\hline$N$ & $\left|\Pi^{\prime}\right|$ & & $\kappa$ & its & $|\Pi|$ & $\# \mathcal{E}_{e v p}$ & $\kappa$ & its & $|\Pi|$ & $\kappa$ & its & $|\Pi|$ \\
\hline \multirow{3}{*}{$3^{3}$} & & a) & 8.68 & 32 & 699 & $2(2.0 \%)$ & & & & & & \\
\hline & 960 & b) & 8.68 & 32 & 699 & $1(1.0 \%)$ & 8.68 & 32 & 699 & $1.37 \mathrm{e}+06$ & 77 & 243 \\
\hline & & c) & 8.69 & 32 & 450 & $1(1.0 \%)$ & & & & & & \\
\hline \multirow{3}{*}{$5^{3}$} & & a) & 9.22 & 33 & 3675 & $25(4.2 \%)$ & & & & & & \\
\hline & 5433 & b) & 9.22 & 33 & 3675 & $12(2.1 \%)$ & 9.22 & 33 & 3669 & $5.53 e+05$ & 216 & 1242 \\
\hline & & c) & 10.53 & 34 & 2325 & $12(2.1 \%)$ & & & & & & \\
\hline \multirow{3}{*}{$7^{3}$} & & a) & 10.84 & 35 & 10101 & $65(3.6 \%)$ & & & & & & \\
\hline & 16248 & b) & 10.84 & 35 & 10101 & $27(1.5 \%)$ & 10.84 & 35 & 10089 & $1.22 \mathrm{e}+06$ & 492 & 3606 \\
\hline & & c) & 13.44 & 38 & 6693 & $27(1.5 \%)$ & & & & & & \\
\hline
\end{tabular}

LOBPCG with up to 200 iterations. We use a stopping criterion of 1e-05 for LOBPCG which, in combination with badly conditioned local matrices, already lead to instability of the solver. The implementation of LOBPCG already states that "excessively small requested tolerance may result in often restarts and instability"; see [42]. However, it should be noted that convergence does not seem to be necessary since $2-5$ iterations already seem to give a stable algorithm with fast convergence.

5.2. Scaling comparisons. In this section, we compare the performance of the algorithms introduced above with four different kinds of scalings. Besides to $\rho$-scaling for which we already gave performance results before, we also test deluxe-, stiffness/K-, and multiplicityscaling. As already reported in [28], neither Algorithm II nor III are sufficient to ensure convergence with $\rho$-scaling. But also for the other scalings as deluxe, Algorithm II is not robust. Concerning Algorithm Ia-c, as expected, the coarse space for deluxe-scaling is the smallest and the fewest iterations are needed for convergence. But deluxe-scaling is also far more costly than $\rho$ - or stiffness-scaling, and these two only need an about 10-15\% larger coarse space to achieve similar convergence results. Multiplicity-scaling cannot be recommended since it uses a coarse space that is almost two times as large as that of deluxe-scaling, i.e., an overhead of $70-90 \%$ is needed.

5.2.1. Composite material. First, we test the four different scalings for the composite material and $1 / h=6 N^{1 / 3}$ as in Section 5.1.1; cf. Table 5.6 for the different scaling results.

5.2.2. Randomly distributed coefficients. Second, we test the four different scalings for randomly distributed coefficients with $20 \%$ high and $80 \%$ low coefficients as in Section 5.1.3 but with $1 / h=8 N^{1 / 3}$ instead of $1 / h=5 N^{1 / 3}$; cf. Tables 5.7 and 5.8 for the different scaling results. Again, Algorithm II and III are not robust for any scaling. Considering Algorithm Ia-c, 


\section{ETNA}

Kent State University and

Johann Radon Institute (RICAM)

ADAPTIVE FETI-DP AND BDDC WITH A GENERALIZED TRANSF. OF BASIS

TABLE 5.3

Compressible linear elasticity. Coarse spaces for $T O L=10$ for all generalized eigenvalue problems.

Representative Volume Element with $\mathrm{E}_{1}=210, \mathrm{E}_{2}=210000$, regular and irregular partitioning, $N=8^{3}$ and $1 / h=32$.

\begin{tabular}{|c|c|c|c|c|c|c|c|c|c|c|c|}
\hline \multicolumn{12}{|c|}{ FETI-DP } \\
\hline & \multicolumn{5}{|c|}{ Algorithm Ia, Ib, and Ic } & \multicolumn{3}{|c|}{ Algorithm II } & \multicolumn{3}{|c|}{ Algorithm III } \\
\hline$\left|\Pi^{\prime}\right|$ & & $\kappa$ & its & $|\Pi|$ & $\mathcal{E}_{\text {evp }}$ & $\kappa$ & its & $|\Pi|$ & $\kappa$ & its & $|\Pi|$ \\
\hline \multirow{3}{*}{18888} & a) & 13.75 & 37 & 1275 & $114(5.6 \%)$ & \multirow{3}{*}{13.75} & \multirow{3}{*}{37} & \multirow{3}{*}{1263} & \multirow{3}{*}{354.30} & \multirow{3}{*}{98} & \multirow{3}{*}{699} \\
\hline & b) & 13.75 & 37 & 1275 & $27(1.4 \%)$ & & & & & & \\
\hline & c) & 13.75 & 38 & 990 & $27(1.4 \%)$ & & & & & & \\
\hline \multicolumn{12}{|c|}{ BDDC } \\
\hline & \multicolumn{5}{|c|}{ Algorithm Ia, Ib, and Ic } & \multicolumn{3}{|c|}{ Algorithm II } & \multicolumn{3}{|c|}{ Algorithm III } \\
\hline$\left|\Pi^{\prime}\right|$ & & $\kappa$ & its & $|\Pi|$ & $\mathcal{E}_{e v p}$ & $\kappa$ & its & $|\Pi|$ & $\kappa$ & its & $|\Pi|$ \\
\hline \multirow{3}{*}{18888} & a) & 13.94 & 31 & 1275 & $114(5.6 \%)$ & \multirow{3}{*}{13.94} & \multirow{3}{*}{31} & \multirow{3}{*}{1263} & \multirow{3}{*}{359.20} & \multirow{3}{*}{84} & \multirow{3}{*}{699} \\
\hline & b) & 13.94 & 31 & 1275 & $27(1.4 \%)$ & & & & & & \\
\hline & c) & 13.94 & 33 & 990 & $27(1.4 \%)$ & & & & & & \\
\hline
\end{tabular}
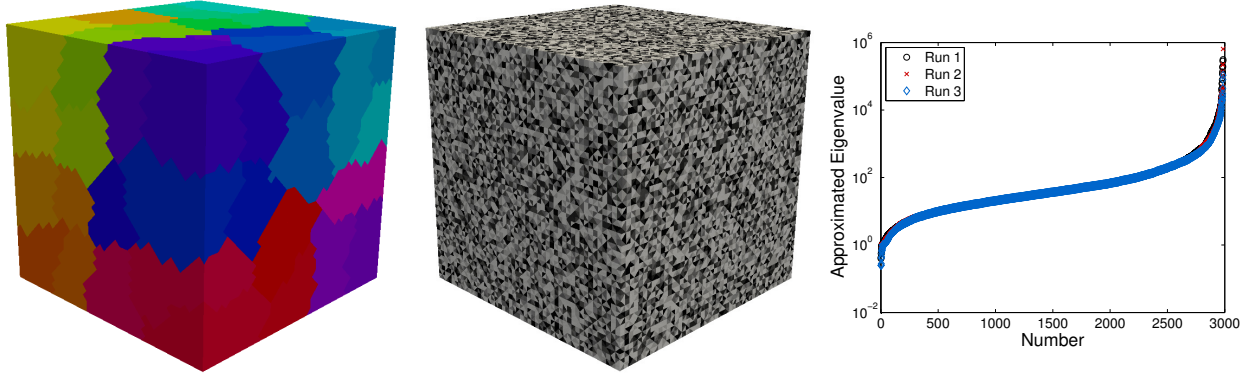

FIGURE 5.3. Irregular decomposition of the unit cube into 64 subdomains (left), randomly distributed coefficients for seven materials with $\mathrm{E}_{1}=1 \mathrm{e}+00$ in lightgray to $\mathrm{E}_{7}=1 \mathrm{e}+06$ in black (center), and approximated local eigenvalues greater 0.1 from all generalized eigenvalue problems (4.1) (right: estimates from two iterations with $L O B P C G$ on the local eigenvalue problems).

the coarse space for deluxe-scaling is again the smallest and the fewest iterations are needed for convergence. But deluxe-scaling is also far more costly than $\rho$ - or stiffness-scaling, and these two only need an about $10-15 \%$ larger coarse space to achieve similar convergence results. Once again, multiplicity-scaling cannot be recommended since it uses a coarse space that is sometimes even two times as large as that of deluxe-scaling.

5.3. Verification of the robustness with LOBPCG eigensolver and randomly distributed coefficients. In this section, we test the LOBPCG eigensolver with blocksize 10, a Cholesky decomposition of the right-hand side of the eigenvalue problem as preconditioner and maximal two iterations on each eigenvalue problem.

We test three different random coefficient distributions for a heterogeneous material composed of seven different homogeneous materials. In these examples, $30 \%$ of the tetrahedra have a Young's Modulus of $\mathrm{E}_{1}=1$, then $20 \%$ have a Young's Modulus of $\mathrm{E}_{2}=10$, and another $10 \%$ each have a Young's Modulus of $\mathrm{E}_{3}=100, \mathrm{E}_{4}=1000, \mathrm{E}_{5}=1 \mathrm{e}+04, \mathrm{E}_{6}=1 \mathrm{e}+05$, and $\mathrm{E}_{7}=1 \mathrm{e}+06$; see Table 5.9. As can be seen from Figure 5.3 (right) the approximated spectrum of the local eigenvalue problems is continuous such that we can expect different results for different tolerances.

We first observe that for all tolerances, convergence is achieved using just two iterations of the LOBPCG eigensolver. Considering the different tolerances, for all runs the approximated condition number is of the size of the chosen tolerance TOL. We state that only TOL $=10$ 


\begin{tabular}{|c|c|c|c|}
\hline$\dot{\omega}_{i}$ & $\omega_{\omega}$ & $\stackrel{D}{\omega}$ & $z$ \\
\hline 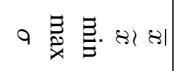 & a $\underset{\tilde{x}}{Z}$ 志. & 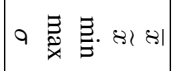 & \\
\hline$\underset{\infty}{\infty}$ & , $\begin{array}{l}\text { Ŭ } \\
\text { c }\end{array}$ & 莣 & 크 \\
\hline $\begin{array}{l}0 \\
\dot{\infty}\end{array}$ & 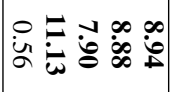 & 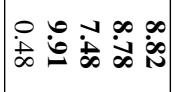 & 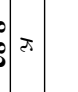 \\
\hline$\sigma \omega$ & $\dot{\dot{u}} \omega$ & $\underset{\omega}{\dot{w}}$ & 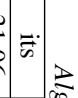 \\
\hline 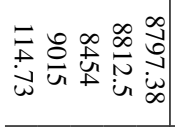 & 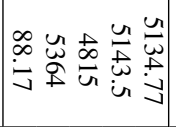 & 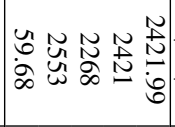 & 年 \\
\hline $\begin{array}{l}u \\
y \\
0 \\
\infty \\
0 \\
a\end{array}$ & 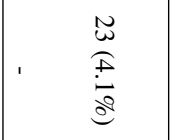 & , $\quad \begin{array}{l}\stackrel{0}{0} \\
\stackrel{\vec{d}}{d}\end{array}$ & $\begin{array}{l}\# \\
\text { \# } \\
0 \\
8 \\
8\end{array}$ \\
\hline 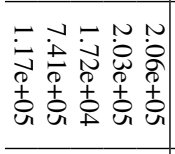 & 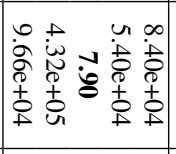 & 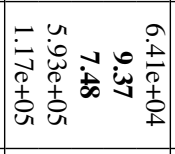 & \\
\hline 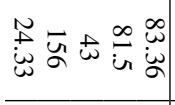 & 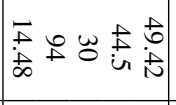 & 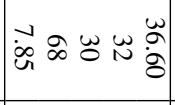 & 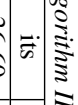 \\
\hline 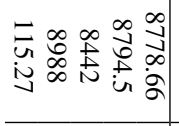 & 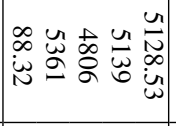 & 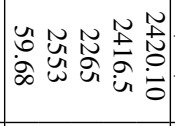 & $=$ \\
\hline 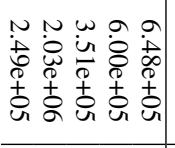 & 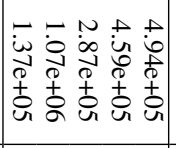 & 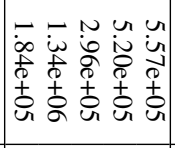 & $\sqrt{2}$ \\
\hline ○ 岁 ŭ & 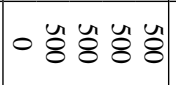 & 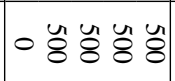 & $\overrightarrow{5}$ \\
\hline 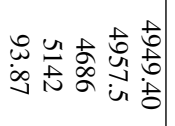 & & $\left\{\begin{array}{l}0 \\
\end{array}\right.$ & $\Xi$ \\
\hline
\end{tabular}

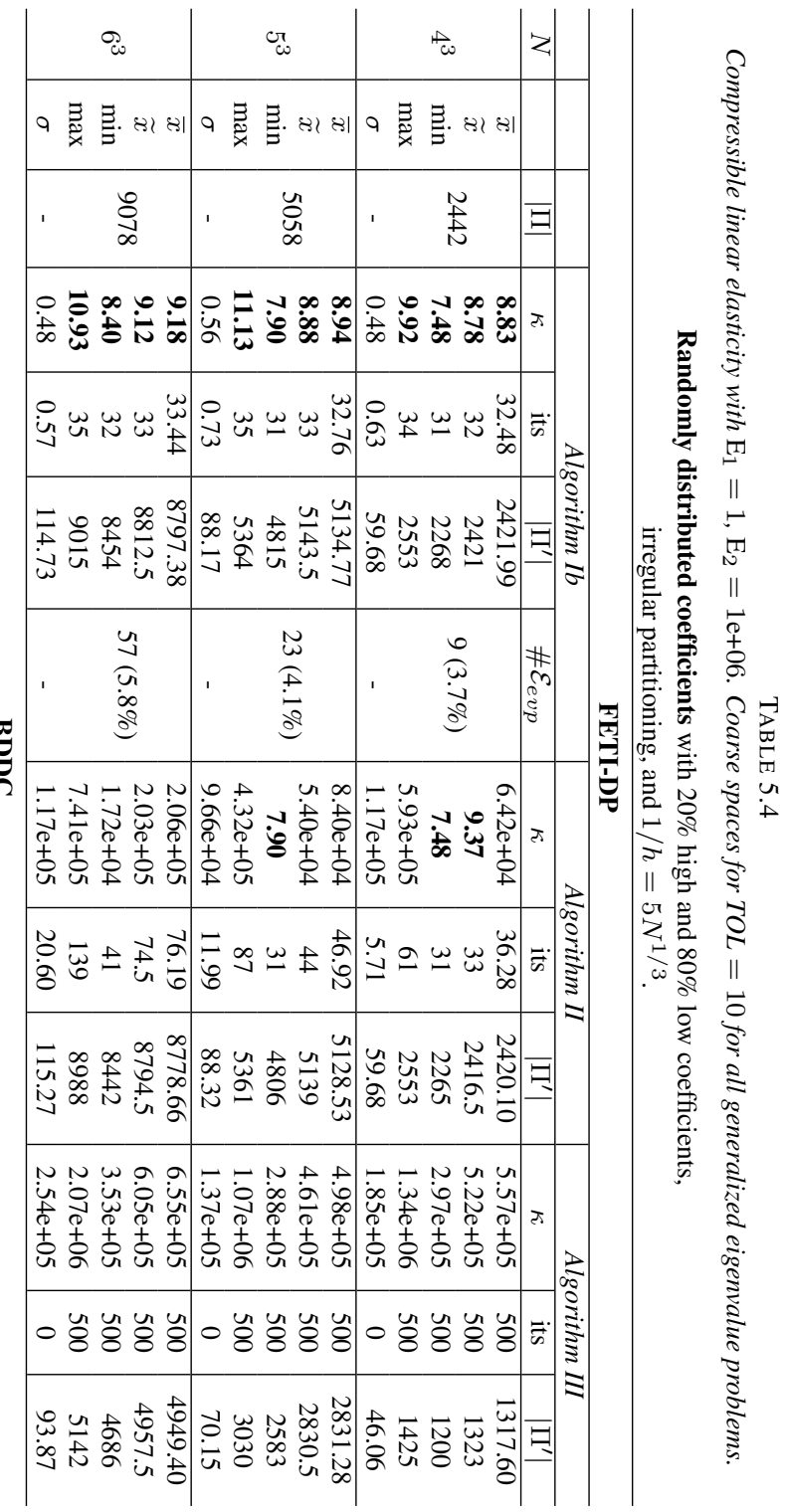




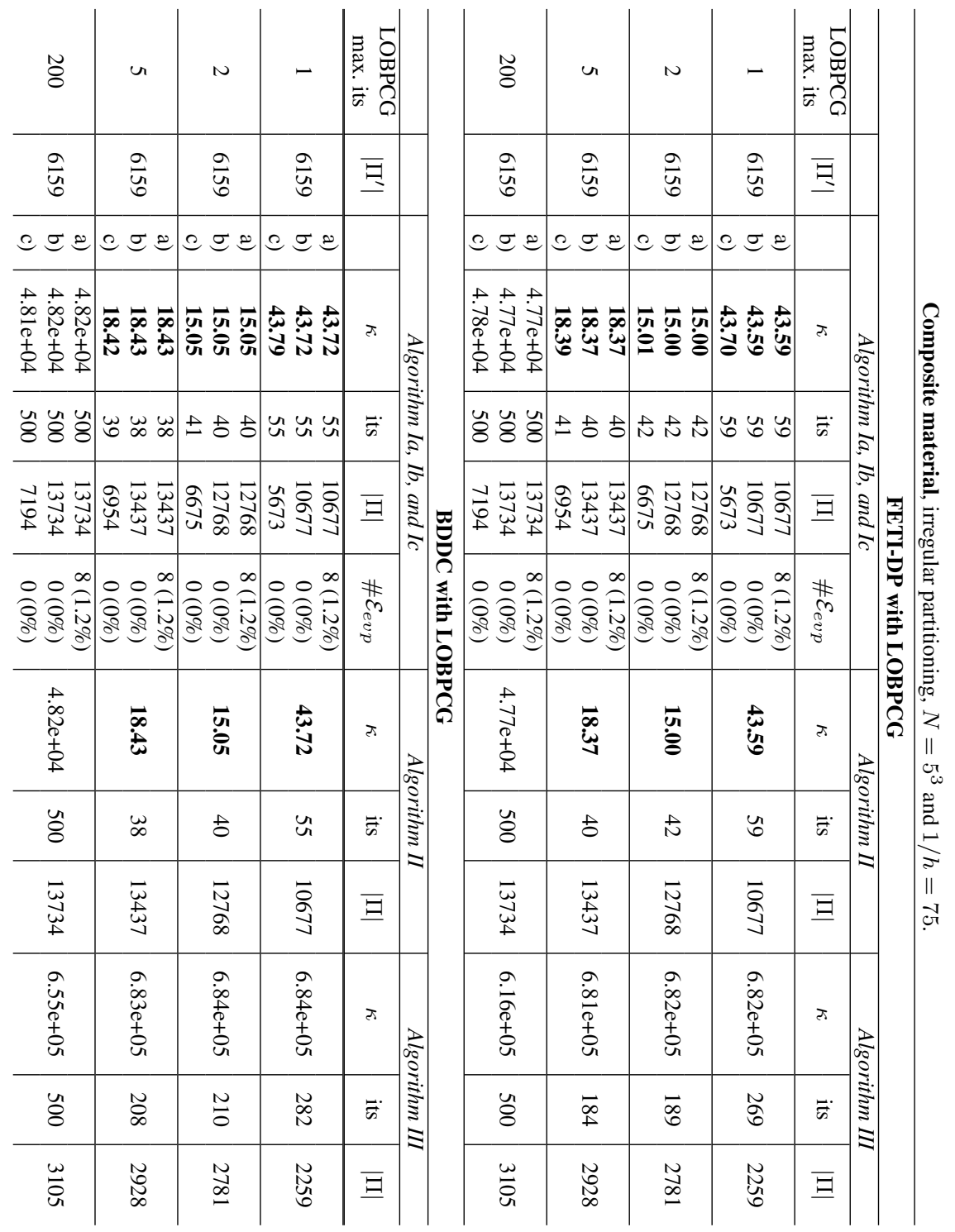




\section{ETNA}

Kent State University and

Johann Radon Institute (RICAM)

TABLE 5.6

Compressible linear elasticity with $\mathrm{E}_{1}=1, \mathrm{E}_{2}=1 \mathrm{e}+06$. Coarse spaces for $\mathrm{TOL}=10$ for all generalized eigenvalue problems.

\begin{tabular}{|c|c|c|c|c|c|c|c|c|c|c|c|c|}
\hline \multicolumn{13}{|c|}{ Composite material, irregular partitioning, and $1 / h=6 N^{1 / 3}$. } \\
\hline \multicolumn{13}{|c|}{$\begin{array}{l}\text { FETI-DP Scaling comparison } \\
\end{array}$} \\
\hline & & \multicolumn{5}{|c|}{ Algorithm Ia, Ib, and Ic } & \multicolumn{3}{|c|}{ Algorithm II } & \multicolumn{3}{|c|}{ Algorithm III } \\
\hline$N$ & $\left|\Pi^{\prime}\right|$ & & $\kappa$ & its & $|\Pi|$ & $\# \mathcal{E}_{\text {evp }}$ & $\kappa$ & its & $|\Pi|$ & $\kappa$ & its & $|\Pi|$ \\
\hline \multicolumn{13}{|c|}{$\rho$-scaling } \\
\hline \multirow{3}{*}{$3^{3}$} & & a) & 8.65 & 34 & 699 & $2(2.0 \%)$ & \multirow{3}{*}{8.65} & \multirow{3}{*}{34} & \multirow{3}{*}{699} & \multirow{3}{*}{$1.36 \mathrm{e}+06$} & \multirow{3}{*}{68} & \multirow{3}{*}{243} \\
\hline & 960 & b) & 8.65 & 34 & 699 & $1(1.0 \%)$ & & & & & & \\
\hline & & c) & 8.67 & 34 & 450 & $1(1.0 \%)$ & & & & & & \\
\hline \multirow{3}{*}{$5^{3}$} & & a) & 9.16 & 35 & 3675 & $25(4.2 \%)$ & \multirow{3}{*}{9.16} & & & & & \\
\hline & 5433 & b) & 9.16 & 35 & 3675 & $12(2.1 \%)$ & & 35 & 3669 & $5.50 \mathrm{e}+05$ & 190 & 1242 \\
\hline & & c) & 10.49 & 36 & 2325 & $12(2.1 \%)$ & & & & & & \\
\hline & & a) & 10.76 & 37 & 10101 & $65(3.6 \%)$ & & & & & & \\
\hline $7^{3}$ & 16248 & b) & 10.76 & 37 & 10101 & $27(1.5 \%)$ & 10.76 & 37 & 10089 & $1.21 \mathrm{e}+06$ & 424 & 3606 \\
\hline & & c) & 13.36 & 39 & 6693 & $27(1.5 \%)$ & & & & & & \\
\hline & & a) & 10.13 & 36 & 19632 & $144(3.6 \%)$ & & & & & & \\
\hline $9^{3}$ & 35838 & b) & 10.13 & 36 & 19632 & $52(1.3 \%)$ & 10.13 & 36 & 19626 & $7.77 e+05$ & 500 & 7053 \\
\hline & & c) & 12.85 & 39 & 12921 & $52(1.3 \%)$ & & & & & & \\
\hline & & & & & & deluxe-s & ling & & & & & \\
\hline & & a) & 7.51 & 20 & 603 & $2(2.0 \%)$ & & & & & & \\
\hline $3^{3}$ & 960 & b) & 7.51 & 20 & 603 & $1(1.0 \%)$ & 7.51 & 20 & 603 & 7.52 & 27 & 207 \\
\hline & & c) & 7.51 & 20 & 393 & $1(1.0 \%)$ & & & & & & \\
\hline & & a) & 9.61 & 29 & 3129 & $25(4.2 \%)$ & & & & & & \\
\hline $5^{3}$ & 5433 & b) & 9.61 & 29 & 3129 & $12(2.1 \%)$ & 9.61 & 29 & 3126 & $9.98 \mathrm{e}+03$ & 77 & 1002 \\
\hline & & c) & 9.63 & 29 & 2004 & $12(2.1 \%)$ & & & & & & \\
\hline & & a) & 7.69 & 30 & 8721 & $65(3.6 \%)$ & & & & & & \\
\hline $7^{3}$ & 16248 & b) & 7.69 & 30 & 8721 & $27(1.5 \%)$ & 7.69 & 30 & 8709 & $3.04 \mathrm{e}+04$ & 178 & 2976 \\
\hline & & c) & 7.70 & 30 & 5859 & $27(1.5 \%)$ & & & & & & \\
\hline & & a) & 10.76 & 34 & 16671 & $144(3.6 \%)$ & & & & & & \\
\hline $9^{3}$ & 35838 & b) & 10.76 & 34 & 16671 & $52(1.3 \%)$ & 10.76 & 34 & 16656 & $8.57 e+04$ & 221 & 5718 \\
\hline & & c) & 10.77 & 34 & 11022 & $52(1.3 \%)$ & & & & & & \\
\hline & & & & & & stiffness- & aling & & & & & \\
\hline & & a) & 7.84 & 26 & 654 & $2(2.0 \%)$ & & & & & & \\
\hline $3^{3}$ & 960 & b) & 7.84 & 26 & 654 & $1(1.0 \%)$ & 7.84 & 26 & 654 & $7.23 e+04$ & 55 & 228 \\
\hline & & c) & 7.85 & 27 & 423 & $1(1.0 \%)$ & & & & & & \\
\hline & & a) & 11.16 & 32 & 3393 & $25(4.2 \%)$ & & & & & & \\
\hline $5^{3}$ & 5433 & b) & 11.16 & 32 & 3393 & $12(2.1 \%)$ & 11.16 & 32 & 3390 & $3.45 \mathrm{e}+04$ & 148 & 1107 \\
\hline & & c) & 11.19 & 32 & 2151 & $12(2.1 \%)$ & & & & & & \\
\hline & & a) & 9.12 & 33 & 9255 & $65(3.6 \%)$ & & & & & & \\
\hline $7^{3}$ & 16248 & b) & 9.12 & 33 & 9255 & $27(1.5 \%)$ & 9.12 & 33 & 9240 & $9.74 \mathrm{e}+04$ & 342 & 3174 \\
\hline & & c) & 9.15 & 34 & 6132 & $27(1.5 \%)$ & & & & & & \\
\hline & & a) & 9.92 & 34 & 17718 & $144(3.6 \%)$ & & & & & & \\
\hline $9^{3}$ & 35838 & b) & 9.92 & 34 & 17718 & $52(1.3 \%)$ & 9.92 & 34 & 17712 & $1.04 \mathrm{e}+05$ & 395 & 6138 \\
\hline & & c) & 9.93 & 34 & 11583 & $52(1.3 \%)$ & & & & & & \\
\hline & & & & & & multiplicit & scaling & & & & & \\
\hline & & a) & 8.63 & 33 & 1029 & $2(2.0 \%)$ & & & & & & \\
\hline $3^{3}$ & 960 & b) & 8.63 & 33 & 1029 & $1(1.01 \%)$ & $5.51 \mathrm{e}+05$ & 54 & 1026 & $1.36 \mathrm{e}+06$ & 345 & 426 \\
\hline & & c) & 8.66 & 35 & 696 & $1(1.01 \%)$ & & & & & & \\
\hline & & a) & 9.10 & 35 & 5172 & $25(4.2 \%)$ & & & & & & \\
\hline $5^{3}$ & 5433 & b) & 9.10 & 35 & 5172 & $12(2.07 \%)$ & 9.10 & 35 & 5169 & $1.62 \mathrm{e}+06$ & 500 & 2115 \\
\hline & & c) & 10.46 & 36 & 3420 & $12(2.07 \%)$ & & & & & & \\
\hline & & a) & 10.73 & 37 & 14625 & $65(3.6 \%)$ & & & & & & \\
\hline $7^{3}$ & 16248 & b) & 10.73 & 37 & 14625 & $27(1.54 \%)$ & 10.73 & 37 & 14619 & $1.72 \mathrm{e}+06$ & 500 & 6183 \\
\hline & & c) & 13.34 & 39 & 10023 & $27(1.54 \%)$ & & & & & & \\
\hline & & a) & 10.09 & 36 & 29598 & $144(3.6 \%)$ & & & & & & \\
\hline $9^{3}$ & 35838 & b) & 10.09 & 36 & 29598 & $52(1.3 \%)$ & 10.09 & 36 & 29592 & $1.58 \mathrm{e}+06$ & 500 & 12477 \\
\hline & & c) & 12.77 & 39 & 20010 & $52(1.3 \%)$ & & & & & & \\
\hline
\end{tabular}




\section{ETNA}

Kent State University and

Johann Radon Institute (RICAM)

TABLE 5.7

Compressible linear elasticity with $\mathrm{E}_{1}=1, \mathrm{E}_{2}=1 \mathrm{e}+06$. Coarse spaces for $T O L=10$ for all generalized eigenvalue problems.

Randomly distributed coefficients with $20 \%$ high and $80 \%$ low coefficients,

irregular partitioning, $N=4^{3}$, and $1 / h=32$.

FETI-DP Scaling comparison; Part 1 ( $\rho$ and deluxe)

\begin{tabular}{|c|c|c|c|c|c|c|c|c|c|c|c|c|}
\hline & & \multicolumn{5}{|c|}{ Algorithm Ia, Ib, and Ic } & \multicolumn{3}{|c|}{ Algorithm II } & \multicolumn{3}{|c|}{ Algorithm III } \\
\hline run & $\left|\Pi^{\prime}\right|$ & & $\kappa$ & its & $|\Pi|$ & $\# \mathcal{E}_{\text {evp }}$ & \begin{tabular}{l|l|}
$\kappa$ \\
\end{tabular} & its & $|\Pi|$ & $\kappa$ & its & $|\Pi|$ \\
\hline \multicolumn{13}{|c|}{$\rho$-scaling } \\
\hline & & a) & 9.24 & 33 & 7350 & $8(2.8 \%)$ & & & & & & \\
\hline \multirow[t]{2}{*}{1} & 2646 & b) & 9.24 & 33 & 7350 & $8(2.8 \%)$ & 12.21 & 34 & 7347 & $5.00 \mathrm{e}+05$ & 500 & 3543 \\
\hline & & c) & 9.24 & 33 & 7350 & $8(2.8 \%)$ & & & & & & \\
\hline \multirow{3}{*}{2} & & a) & 9.40 & 33 & 7278 & $8(2.8 \%)$ & & & & & & \\
\hline & 2646 & b) & 9.40 & 33 & 7278 & $8(2.8 \%)$ & $1.57 \mathrm{e}+0 \$$ & 52 & 7272 & $4.74 e+05$ & 500 & 3480 \\
\hline & & c) & 9.40 & 33 & 7278 & $8(2.8 \%)$ & & & & & & \\
\hline \multirow{3}{*}{3} & & a) & 8.32 & 33 & 7320 & $8(2.8 \%)$ & & & & & & \\
\hline & 2646 & b) & 8.32 & 33 & 7320 & $8(2.8 \%)$ & $6.52 \mathrm{e}+04$ & 67 & 7308 & $4.72 \mathrm{e}+05$ & 500 & 3525 \\
\hline & & c) & 8.32 & 33 & 7320 & $8(2.8 \%)$ & & & & & & \\
\hline \multirow{3}{*}{4} & & a) & 9.44 & 34 & 7230 & $8(2.8 \%)$ & & & & & & \\
\hline & 2646 & b) & 9.44 & 34 & 7230 & $8(2.8 \%)$ & 9.44 & 34 & 7227 & $4.67 e+05$ & 500 & 3408 \\
\hline & & c) & 9.44 & 34 & 7230 & $8(2.8 \%)$ & & & & & & \\
\hline \multirow{3}{*}{5} & & a) & 9.26 & 33 & 7416 & $8(2.8 \%)$ & & & & & & \\
\hline & 2646 & b) & 9.26 & 33 & 7416 & $8(2.8 \%)$ & $2.54 \mathrm{e}+0 \$$ & 73 & 7407 & $4.69 e+05$ & 500 & 3588 \\
\hline & & c) & 9.26 & 33 & 7416 & $8(2.8 \%)$ & & & & & & \\
\hline \multirow{3}{*}{6} & & a) & 9.73 & 34 & 7311 & $8(2.8 \%)$ & & & & & & \\
\hline & 2646 & b) & 9.73 & 34 & 7311 & $8(2.8 \%)$ & 9.73 & 34 & 7308 & $5.80 \mathrm{e}+05$ & 500 & 3477 \\
\hline & & c) & 9.73 & 34 & 7311 & $8(2.8 \%)$ & & & & & & \\
\hline \multicolumn{13}{|c|}{ deluxe-scaling } \\
\hline \multirow{3}{*}{1} & & a) & 6.08 & 21 & 6174 & $8(2.8 \%)$ & & & & & & \\
\hline & 2646 & b) & 6.08 & 21 & 6174 & $8(2.8 \%)$ & 7.09 & 22 & 6171 & $1.86 e+05$ & 358 & 2769 \\
\hline & & c) & 6.08 & 21 & 6174 & $8(2.8 \%)$ & & & & & & \\
\hline \multirow{3}{*}{2} & & a) & 5.65 & 22 & 5997 & $8(2.8 \%)$ & & & & & & \\
\hline & 2646 & b) & 5.65 & 22 & 5997 & $8(2.8 \%)$ & 5.65 & 22 & 5997 & $6.90 e+04$ & 285 & 2643 \\
\hline & & c) & 5.65 & 22 & 5997 & $8(2.8 \%)$ & & & & & & \\
\hline \multirow{3}{*}{3} & & a) & 4.89 & 23 & 6069 & $8(2.8 \%)$ & & & & & & \\
\hline & 2646 & b) & 4.89 & 23 & 6069 & $8(2.8 \%)$ & $3.12 \mathrm{e}+04$ & 42 & 6057 & $2.35 e+05$ & 433 & 2703 \\
\hline & & c) & 4.89 & 23 & 6069 & $8(2.8 \%)$ & & & & & & \\
\hline \multirow{3}{*}{4} & & a) & 5.94 & 24 & 5979 & $8(2.8 \%)$ & & & & & & \\
\hline & 2646 & b) & 5.94 & 24 & 5979 & $8(2.8 \%)$ & 5.94 & 24 & 5976 & $2.07 e+05$ & 336 & 2601 \\
\hline & & c) & 5.94 & 24 & 5979 & $8(2.8 \%)$ & & & & & & \\
\hline \multirow{3}{*}{5} & & a) & 4.70 & 22 & 6207 & $8(2.8 \%)$ & & & & & & \\
\hline & 2646 & b) & 4.70 & 22 & 6207 & $8(2.8 \%)$ & $3.14 \mathrm{e}+04$ & 36 & 6201 & $1.37 \mathrm{e}+05$ & 320 & 2799 \\
\hline & & c) & 4.70 & 22 & 6207 & $8(2.8 \%)$ & & & & & & \\
\hline \multirow{3}{*}{6} & & a) & 4.43 & 22 & 6249 & $8(2.8 \%)$ & & & & & & \\
\hline & 2646 & b) & 4.43 & 22 & 6249 & $8(2.8 \%)$ & 4.43 & 22 & 6246 & $1.95 e+05$ & 346 & 2841 \\
\hline & & c) & 4.43 & 22 & 6249 & $8(2.8 \%)$ & & & & & & \\
\hline
\end{tabular}

ensures convergence within less than 50 iterations, but it also uses a coarse space that is three times as large as that of TOL $=100$ and nine to ten times as large as that of TOL $=1000$. A trade-off between fast convergence and a manageable size of the coarse space is a problemand facility-dependent task.

6. Parallel results. We present some preliminary CPU timings using a first parallel implementation of our adaptive FETI-DP Algorithms Ia and Ic compared to the standard nonadaptive FETI-DP with a full vertex and edge average coarse space. In all FETI-DP methods, we use stiffness scaling, that is, we use the diagonal entries of the stiffness matrices. We use PETSc [2, 3] with the PARDISO solver from the Intel MKL $[23,58]$ as a direct solver, and the SLEPc software library $[22,56]$ to solve the local generalized eigenvalue problems. 


\section{ETNA}

Kent State University and

Johann Radon Institute (RICAM)

TABLE 5.8

Compressible linear elasticity with $\mathrm{E}_{1}=1, \mathrm{E}_{2}=1 \mathrm{e}+06$. Coarse spaces for $T O L=10$ for all generalized eigenvalue problems.

Randomly distributed coefficients with $20 \%$ high and $80 \%$ low coefficients,

irregular partitioning, $N=4^{3}$, and $1 / h=32$.

\begin{tabular}{|c|c|c|c|c|c|c|c|c|c|c|c|c|}
\hline \multicolumn{13}{|c|}{ FETI-DP Scaling comparison; Part 2 (stiffness and multiplicity) } \\
\hline & & \multicolumn{5}{|c|}{ Algorithm Ia, Ib, and Ic } & \multicolumn{3}{|c|}{ Algorithm II } & \multicolumn{3}{|c|}{ Algorithm III } \\
\hline run & $\left|\Pi^{\prime}\right|$ & & $\kappa$ & its & $|\Pi|$ & $\# \mathcal{E}_{\text {evp }}$ & $\kappa$ & its & $|\Pi|$ & $\kappa$ & its & $|\Pi|$ \\
\hline \multicolumn{13}{|c|}{ stiffness-scaling } \\
\hline \multirow{3}{*}{1} & & a) & 7.82 & 29 & 6921 & $8(2.8 \%)$ & \multirow{3}{*}{7.82} & \multirow{3}{*}{29} & \multirow{3}{*}{6918} & \multirow{3}{*}{$2.15 \mathrm{e}+05$} & \multirow{3}{*}{500} & \multirow{3}{*}{3177} \\
\hline & 2646 & b) & 7.82 & 29 & 6921 & $8(2.8 \%)$ & & & & & & \\
\hline & & c) & 7.82 & 29 & 6921 & $8(2.8 \%)$ & & & & & & \\
\hline \multirow{3}{*}{2} & & a) & 7.40 & 27 & 6876 & $8(2.8 \%)$ & \multirow{3}{*}{$2.86 e+04$} & \multirow{3}{*}{43} & \multirow{3}{*}{6870} & \multirow{3}{*}{$3.05 e+05$} & \multirow{3}{*}{500} & \multirow{3}{*}{3171} \\
\hline & 2646 & b) & 7.40 & 27 & 6876 & $8(2.8 \%)$ & & & & & & \\
\hline & & c) & 7.40 & 27 & 6876 & $8(2.8 \%)$ & & & & & & \\
\hline \multirow{3}{*}{3} & & a) & 9.97 & 30 & 6903 & $8(2.8 \%)$ & \multirow{3}{*}{$6.47 e+04$} & \multirow{3}{*}{61} & & & & \\
\hline & 2646 & b) & 9.97 & 30 & 6903 & $8(2.8 \%)$ & & & 6891 & $2.72 \mathrm{e}+05$ & 500 & 3195 \\
\hline & & c) & 9.97 & 30 & 6903 & $8(2.8 \%)$ & & & & & & \\
\hline & & a) & 7.80 & 30 & 6738 & $8(2.8 \%)$ & & & & & & \\
\hline 4 & 2646 & b) & 7.80 & 30 & 6738 & $8(2.8 \%)$ & 7.80 & 30 & 6735 & $2.19 \mathrm{e}+05$ & 500 & 3033 \\
\hline & & c) & 7.80 & 30 & 6738 & $8(2.8 \%)$ & & & & & & \\
\hline & & a) & 8.49 & 29 & 6987 & $8(2.8 \%)$ & & & & & & \\
\hline 5 & 2646 & b) & 8.49 & 29 & 6987 & $8(2.8 \%)$ & $3.63 e+04$ & 60 & 6978 & $2.15 \mathrm{e}+05$ & 500 & 3228 \\
\hline & & c) & 8.49 & 29 & 6987 & $8(2.8 \%)$ & & & & & & \\
\hline & & a) & 7.32 & 29 & 6888 & $8(2.8 \%)$ & & & & & & \\
\hline 6 & 2646 & b) & 7.32 & 29 & 6888 & $8(2.8 \%)$ & 7.32 & 29 & 6885 & $3.04 \mathrm{e}+05$ & 500 & 3144 \\
\hline & & c) & 7.32 & 29 & 6888 & $8(2.8 \%)$ & & & & & & \\
\hline & & & & & & multiplic & -scaling & & & & & \\
\hline & & a) & 8.48 & 32 & 12744 & $8(2.8 \%)$ & & & & & & \\
\hline 1 & 2646 & b) & 8.48 & 32 & 12744 & $8(2.8 \%)$ & $6.12 \mathrm{e}+05$ & 116 & 12732 & $9.70 \mathrm{e}+05$ & 500 & 8574 \\
\hline & & c) & 8.48 & 32 & 12744 & $8(2.8 \%)$ & & & & & & \\
\hline & & a) & 9.02 & 33 & 12900 & $8(2.8 \%)$ & & & & & & \\
\hline 2 & 2646 & b) & 9.02 & 33 & 12900 & $8(2.8 \%)$ & $9.69 e+05$ & 130 & 12885 & $1.31 \mathrm{e}+06$ & 500 & 8721 \\
\hline & & c) & 9.02 & 33 & 12900 & $8(2.8 \%)$ & & & & & & \\
\hline & & a) & 9.42 & 33 & 13092 & $8(2.8 \%)$ & & & & & & \\
\hline 3 & 2646 & b) & 9.42 & 33 & 13092 & $8(2.8 \%)$ & $6.32 \mathrm{e}+04$ & 64 & 13080 & $1.42 \mathrm{e}+06$ & & 8922 \\
\hline & & c) & 9.42 & 33 & 13092 & $8(2.8 \%)$ & & & & & & \\
\hline & & a) & 9.13 & 33 & 13074 & $8(2.8 \%)$ & & & & & & \\
\hline 4 & 2646 & b) & 9.13 & 33 & 13074 & $8(2.8 \%)$ & $2.69 \mathrm{e}+05$ & 140 & 13059 & $1.35 e+06$ & 500 & 8895 \\
\hline & & c) & 9.13 & 33 & 13074 & $8(2.8 \%)$ & & & & & & \\
\hline & & a) & 7.78 & 31 & 12972 & $8(2.8 \%)$ & & & & & & \\
\hline 5 & 2646 & b) & 7.78 & 31 & 12972 & $8(2.8 \%)$ & $2.48 \mathrm{e}+05$ & 67 & 12963 & $1.85 \mathrm{e}+06$ & 500 & 8805 \\
\hline & & c) & 7.78 & 31 & 12972 & $8(2.8 \%)$ & & & & & & \\
\hline & & a) & 8.82 & 32 & 12894 & $8(2.8 \%)$ & & & & & & \\
\hline 6 & 2646 & b) & 8.82 & 32 & 12894 & $8(2.8 \%)$ & $3.18 \mathrm{e}+05$ & 104 & 12882 & $1.11 \mathrm{e}+06$ & 500 & 8718 \\
\hline & & c) & 8.82 & 32 & 12894 & $8(2.8 \%)$ & & & & & & \\
\hline
\end{tabular}

As before, we consider the unit cube and the linear elastic composite material as depicted in Figure 5.1 (left) with a METIS decomposition. We enforce zero Dirichlet boundary conditions on the face with $x=0$ and apply the body force $f=(0.1,0.1,0.1)^{T}$. We use conforming $\mathcal{P}_{2}$ finite elements on a mesh with $H / h=6$.

For Algorithm Ia, the eigenvalue problems are solved by the SLEPc Krylov-Schur method with block size 10. In Algorithm Ic, we use only two iterations of the Krylov-Schur method with block size 10 .

In our parallel implementation, we make the more realistic assumption that the coefficient evaluation function is not known. Thus, the strategy of Algorithm Ic, to discard eigenvalue problems and constraints, is based on the stiffness scaling from which we try to deduce coefficient jumps. We also use TOL $=50 \log (H / h)$ to reduce the adaptive coarse space 
TABLE 5.9

Compressible linear elasticity for a heterogeneous material composed of seven different homogeneous materials with $\mathrm{E}_{1}=1$ to $\mathrm{E}_{7}=1 \mathrm{e}+06$. Coarse spaces for $T O L=10, T O L=100$, and $T O L=1000$ for all generalized eigenvalue problems. Solution of the local eigenvalue problems by LOBPCG with blocksize 10 and a maximum of two iterations.

\begin{tabular}{|c|c|c|c|c|c|c|c|c|c|c|c|c|}
\hline \multirow[b]{2}{*}{ run } & \multirow[b]{2}{*}{$\left|\Pi^{\prime}\right|$} & \multicolumn{5}{|c|}{ Algorithm Ia, Ib, and Ic } & \multicolumn{3}{|c|}{ Algorithm II } & \multicolumn{3}{|c|}{ Algorithm III } \\
\hline & & & $\kappa$ & its & $|\Pi|$ & $\# \mathcal{E}_{e v p}$ & $\kappa$ & its & $|\Pi|$ & $\kappa$ & its & $|\Pi|$ \\
\hline \multicolumn{13}{|c|}{$\mathrm{TOL}=10$} \\
\hline \multirow{3}{*}{1} & & a) & 14.11 & 41 & 12084 & $2(0.7 \%)$ & \multirow{3}{*}{14.11} & \multirow{3}{*}{41} & \multirow{3}{*}{12084} & \multirow{3}{*}{$1.12 \mathrm{e}+05$} & \multirow{3}{*}{500} & \multirow{3}{*}{4842} \\
\hline & 2820 & b) & 14.11 & 41 & 12084 & $2(0.7 \%)$ & & & & & & \\
\hline & & c) & 14.11 & 41 & 12084 & $2(0.7 \%)$ & & & & & & \\
\hline \multirow{3}{*}{2} & & a) & 13.18 & 40 & 12186 & $2(0.7 \%)$ & \multirow{3}{*}{13.18} & \multirow{3}{*}{40} & \multirow{3}{*}{12186} & \multirow{3}{*}{$2.12 \mathrm{e}+05$} & \multirow{3}{*}{500} & \multirow{3}{*}{4920} \\
\hline & 2820 & b) & 13.18 & 40 & 12186 & $2(0.7 \%)$ & & & & & & \\
\hline & & c) & 13.18 & 40 & 12186 & $2(0.7 \%)$ & & & & & & \\
\hline \multirow{3}{*}{3} & & a) & 18.89 & 42 & 12147 & $2(0.7 \%)$ & \multirow{3}{*}{18.89} & \multirow{3}{*}{42} & \multirow{3}{*}{12147} & \multirow{3}{*}{$1.04 \mathrm{e}+05$} & \multirow{3}{*}{500} & \multirow{3}{*}{4863} \\
\hline & 2820 & b) & 18.89 & 42 & 12147 & $2(0.7 \%)$ & & & & & & \\
\hline & & c) & 18.89 & 42 & 12147 & $2(0.7 \%)$ & & & & & & \\
\hline & & & & & & TOL & & & & & & \\
\hline & & a) & 107.26 & 113 & 4278 & $2(0.7 \%)$ & & & & & & \\
\hline 1 & 2820 & b) & 107.26 & 113 & 4278 & $2(0.7 \%)$ & 107.26 & 113 & 4278 & $1.11 \mathrm{e}+05$ & 500 & 987 \\
\hline & & c) & 107.26 & 113 & 4278 & $2(0.7 \%)$ & & & & & & \\
\hline & & a) & 100.32 & 110 & 4299 & $2(0.7 \%)$ & & & & & & \\
\hline 2 & 2820 & b) & 100.32 & 110 & 4299 & $2(0.7 \%)$ & 100.32 & 110 & 4299 & $2.11 \mathrm{e}+05$ & 500 & 1041 \\
\hline & & c) & 100.32 & 110 & 4299 & $2(0.7 \%)$ & & & & & & \\
\hline & & a) & 115.62 & 114 & 4329 & $2(0.7 \%)$ & & & & & & \\
\hline 3 & 2820 & b) & 115.62 & 114 & 4329 & $2(0.7 \%)$ & 115.62 & 114 & 4329 & $1.04 \mathrm{e}+05$ & 500 & 1041 \\
\hline & & c) & 115.62 & 114 & 4329 & $2(0.7 \%)$ & & & & & & \\
\hline & & & & & & $\mathrm{TOL}=$ & & & & & & \\
\hline & & a) & 970.55 & 321 & 1311 & $2(0.7 \%)$ & & & & & & \\
\hline 1 & 2820 & b) & 970.55 & 321 & 1311 & $2(0.7 \%)$ & 970.55 & 321 & 1311 & $1.11 \mathrm{e}+05$ & 500 & 321 \\
\hline & & c) & 970.55 & 321 & 1311 & $2(0.7 \%)$ & & & & & & \\
\hline & & a) & 993.08 & 320 & 1260 & $2(0.7 \%)$ & & & & & & \\
\hline 2 & 2820 & b) & 993.08 & 320 & 1260 & $2(0.7 \%)$ & 993.08 & 320 & 1260 & $2.08 \mathrm{e}+05$ & 500 & 318 \\
\hline & & c) & 993.08 & 320 & 1260 & $2(0.7 \%)$ & & & & & & \\
\hline & & a) & 1609.10 & 343 & 1158 & $2(0.7 \%)$ & & & & & & \\
\hline 3 & 2820 & b) & 1609.10 & 343 & 1158 & $2(0.7 \%)$ & 1609.10 & 343 & 1158 & $1.03 e+05$ & 500 & 273 \\
\hline & & c) & 1609.10 & 343 & 1158 & $2(0.7 \%)$ & & & & & & \\
\hline
\end{tabular}

dimension; cf. Table 5.9 for a comparison of different tolerances. If the local iterative solver breaks down due to the high condition numbers of the local matrices, then we use the SLEPc interface to the LAPACK solver [1].

We also report the size of the coarse space (nonadaptive and adaptive) denoted by $|\widehat{\Pi}|$ and the number of nonzeros in the coarse space matrix in parentheses. The computation is conducted with one subdomain per core on the supercomputer magnitUDE at the Center for Computational Sciences and Simulation (CCSS) of the University of Duisburg-Essen. We use a preconditioned conjugate gradients method with up to 10,000 iterations and a maximum wall time of one hour. We report the number of Krylov iterations, the total runtime, and the KSP residual norm $\|r e s\|$ at convergence or cancellation when the wall time is exceeded.

Table 6.1 shows that the standard nonadaptive method does not converge as required and is thus not suited for this problem. The adaptive methods reduce the run time significantly and also reduce the residual to the given tolerance. For this problem and the strategy of Algorithm Ic, we obtain comparable results for both adaptive methods. 


\section{ETNA}

Kent State University and

Johann Radon Institute (RICAM)

TABLE 6.1

Compressible linear elasticity with $\mathrm{E}_{1}=1, \mathrm{E}_{2}=1 \mathrm{e}+06$. Coarse spaces for $T O L=50 \log (H / h)$ for all generalized eigenvalue problems.

Composite material

irregular partitioning, and $H / h=6$.

\begin{tabular}{|c|c|c|c|c|c|c|c|c|}
\hline & \multicolumn{4}{|c|}{$N=64$} & \multicolumn{4}{|c|}{$N=216$} \\
\hline & its & $\|r e s\|$ & runtime & $\begin{array}{c}|\widehat{\Pi}| \\
(\mathrm{nnz})\end{array}$ & its & $\|r e s\|$ & runtime & $\begin{array}{c}|\widehat{\Pi}| \\
(\mathrm{nnz})\end{array}$ \\
\hline $\begin{array}{l}\text { Standard } \\
\text { FETI-DP }\end{array}$ & $>6373$ & $5.22 \mathrm{e}-5$ & $>60 \mathrm{~min}$ & $\begin{array}{c}2346 \\
(859,212) \\
\end{array}$ & $>6033$ & $7.56 \mathrm{e}-4$ & $>60 \mathrm{~min}$ & $\begin{array}{c}9828 \\
(4,466,412)\end{array}$ \\
\hline $\begin{array}{l}\text { Adaptive } \\
\text { FETI-DP } \\
\text { (Alg. Ia) }\end{array}$ & 63 & $4.68 \mathrm{e}-11$ & $23.4 \mathrm{~min}$ & $\begin{array}{c}3258 \\
(1,379,072) \\
\end{array}$ & 75 & $8.83 e-11$ & $37.9 \mathrm{~min}$ & $\begin{array}{c}13627 \\
(7,393,571)\end{array}$ \\
\hline $\begin{array}{l}\text { Adaptive } \\
\text { FETI-DP } \\
\text { (Alg. Ic) }\end{array}$ & 63 & $4.30 \mathrm{e}-11$ & $21.7 \mathrm{~min}$ & $\begin{array}{c}3248 \\
(1,374,056)\end{array}$ & 76 & $5.85 \mathrm{e}-11$ & $35.7 \mathrm{~min}$ & $\begin{array}{c}13615 \\
(7,384,271)\end{array}$ \\
\hline
\end{tabular}

7. Conclusion. In [28], an adaptive coarse space for FETI-DP domain decomposition methods applied to heterogeneous elliptic problems in three dimension was introduced. The method is based on numerically solving local eigenvalue problems on faces and edges of subdomains and on using these eigenvectors as deflation vectors. The condition number of the resulting preconditioner operator using deflation is bounded independently of the heterogeneity. Then, in [30], for heterogeneous problems and general scalings, a correspondence was shown between FETI-DP methods using deflation and FETI-DP and BDDC methods using a generalized transformation of basis combined with a partial finite element assembly.

In this current paper, we now combine the approaches in [28] and [30] and obtain FETI-DP and BDDC methods with a condition number bound independent of heterogeneities but using a generalized transformation of basis instead of deflation. For the new approach, it will be easier to extend the parallel scalability to a large number of subdomains on large supercomputers, also for heterogeneous problems, by solving the coarse problem inexactly. This is not possible in projection approaches like deflation or balancing.

We also present comparisons of the adaptive method with different scalings such as $\rho$-, deluxe-, stiffness-, and multiplicity-scaling. For our test cases, we state that $\rho$-scaling just needs about $10 \%$ of additional constraints compared to deluxe-scaling. The findings of stiffness-scaling are comparable to those of $\rho$-scaling. Multiplicity-scaling on the other hand gives significantly larger coarse spaces.

We additionally show that, also for hard problems including those with random coefficients with seven different materials, only two iterations of an iterative solver on the local eigenvalue problems can be sufficient to obtain fast convergence of the overall method.

Eventually, we present some preliminary results for parallel adaptive FETI-DP to show that Algorithms Ia and Ic both excel the standard nonadaptive approach with respect to run time and residual norm reduction.

Acknowledgements. The authors gratefully acknowledge the computing time granted by the Center for Computational Sciences and Simulation (CCSS) of the University of DuisburgEssen and provided on the supercomputer magnitUDE (DFG grants INST 20876/209-1 FUGG, INST 20876/243-1 FUGG) at the Zentrum für Informations- und Mediendienste (ZIM).

\section{REFERENCES}

[1] E. Anderson, Z. Bai, C. H. Bischof, L. S. Blackford, J. W. Demmel, J. J. Dongarra, J. Du Croz, A. Greenbaum, S. J. Hammarling, A. McKenney, and D. Sorensen, LAPACK Users' Guide, 3rd ed., SIAM, Philadelphia, 1999. 
[2] S. Balay, S. Abhyankar, M. F. Adams, J. Brown, P. Brune, K. Buschelman, L. Dalcin, V. Eijkhout, W. D. Gropp, D. KaushiK, M. G. Knepley, L. C. Mcinnes, K. Rupp, B. F. SMith, S. Zampini, AND H. Zhang, PETSc users manual, Tech. Rep. ANL-95/11-Revision 3.8, Mathematics and Computer Science Division, Argonne National Laboratory, Argonne, 2017.

[3] S. Balay, W. D. Gropp, L. C. MCInnes, And B. F. SMith, Efficient management of parallelism in object oriented numerical software libraries, in Modern Software Tools in Scientific Computing, E. Arge, A. M. Bruaset, and H. P. Langtangen, eds., Birkhäuser, Basel, 1997, pp. 163-202.

[4] L. Beirão da Veiga, L. F. PAVARINo, S. SCACChI, O. B. Widlund, AND S. ZAMPIni, Isogeometric BDDC preconditioners with deluxe scaling, SIAM J. Sci. Comput., 36 (2014), pp. A1118-A1139.

[5] - Adaptive selection of primal constraints for isogeometric BDDC deluxe preconditioners, SIAM J. Sci. Comput., 39 (2017), pp. A281-A302.

[6] P. BJøRSTAD, J. Koster, AND P. KRZYŻANOWSKi, Domain decomposition solvers for large scale industrial finite element problems, in Applied Parallel Computing. New Paradigms for HPC in Industry and Academia, T. Sørevik, F. Manne, A. H. Gebremedhin, and R. Moe, eds., vol. 1947 of Lecture Notes in Comput. Sci., Springer, Berlin, 2001, pp. 373-383.

[7] P. BJøRSTAD AND P. KRZYŻANOWSKI, A flexible 2-level Neumann-Neumann method for structural analysis problems, in Parallel Processing and Applied Mathematics, R. Wyrzykowski, J. Dongarra, M. Paprzycki, and J. Waśniewski, eds., vol. 2328 of Lecture Notes in Comput. Sci., Springer, Berlin, 2002, pp. 387-394.

[8] J. G. CALVO, A BDDC algorithm with deluxe scaling for $H$ (curl) in two dimensions with irregular subdomains, Math. Comp., 85 (2016), pp. 1085-1111.

[9] J. G. CALVO AND O. B. WIDLUND, An adaptive choice of primal constraints for BDDC domain decomposition algorithms, Electron. Trans. Numer. Anal., 45 (2016), pp. 524-544. http://etna.ricam.oeaw.ac.at/vol.45.2016/pp524-544.dir/pp524-544.pdf

[10] E. T. CHUNG AND H. H. KIM, A deluxe FETI-DP algorithm for a hybrid staggered discontinuous Galerkin method for H(curl)-elliptic problems, Internat. J. Numer. Methods Engrg., 98 (2014), pp. 1-23.

[11] J.-M. CRos, A preconditioner for the Schur complement domain decomposition method, in Domain Decomposition Methods in Science and Engineering, I. Herrera, D. E. Keyes, O. B. Widlund, R. Yates, eds., National Autonomous University of Mexico (UNAM), Mexico City, 2003, pp. 373-380.

[12] C. Dohrmann AND C. PeChSTEIn, Modern domain decomposition solvers - BDDC, deluxe scaling, and an algebraic approach, Talk by C. Pechstein at the University Linz, December 2013. http://people.ricam.oeaw.ac.at/c.pechstein/pechstein-bddc2013.pdf

[13] C. R. DOHRMANN, A preconditioner for substructuring based on constrained energy minimization, SIAM J. Sci. Comput., 25 (2003), pp. 246-258.

[14] C. R. Dohrmann AND O. B. WIDLund, Some recent tools and a BDDC algorithm for 3D problems in $H$ (curl), in Domain Decomposition Methods in Science and Engineering XX, R. Bank, M. Holst, O. Widlund, and J. Xu, eds., vol. 91 of Lect. Notes Comput. Sci. Eng., Springer, Heidelberg, 2013, pp. $15-25$.

[15] V. Dolean, F. Nataf, R. Scheichl, And N. Spillane, Analysis of a two-level Schwarz method with coarse spaces based on local Dirichlet-to-Neumann maps, Comput. Methods Appl. Math., 12 (2012), pp. 391-414.

[16] Y. EfEndiev, J. Galvis, R. LAZARov, AND J. Willems, Robust domain decomposition preconditioners for abstract symmetric positive definite bilinear forms, ESAIM Math. Model. Numer. Anal., 46 (2012), pp. 1175-1199.

[17] C. Farhat, M. Lesoinne, P. LeTallec, K. Pierson, and D. Rixen, FEti-DP: a dual-primal unified FETI method. I. A faster alternative to the two-level FETI method, Internat. J. Numer. Methods Engrg., 50 (2001), pp. 1523-1544.

[18] C. FARHAT, M. Lesoinne, AND K. Pierson, A scalable dual-primal domain decomposition method, Numer. Linear Algebra Appl., 7 (2000), pp. 687-714.

[19] Y. FRAGAKIS AND M. PAPADRAKAKIS, The mosaic of high performance domain decomposition methods for structural mechanics: formulation, interrelation and numerical efficiency of primal and dual methods, Comput. Methods Appl. Mech. Engrg., 192 (2003), pp. 3799-3830.

[20] J. GALVIS AND Y. EFENDIEv, Domain decomposition preconditioners for multiscale flows in high-contrast media, Multiscale Model. Simul., 8 (2010), pp. 1461-1483.

[21] _ Domain decomposition preconditioners for multiscale flows in high contrast media: reduced dimension coarse spaces, Multiscale Model. Simul., 8 (2010), pp. 1621-1644.

[22] V. HeRnANDEZ, J. E. ROMAN, AND V. VidAL, SLEPc: a scalable and flexible toolkit for the solution of eigenvalue problems, ACM Trans. Math. Software, 31 (2005), pp. 351-362.

[23] INTEL, Intel Math Kernel Library developer reference, 2015. https://software.intel.com/en-us/articles/mkl-reference-manual

[24] M. Jarošová, A. Klawonn, AND O. RheinbaCh, Projector preconditioning and transformation of basis in FETI-DP algorithms for contact problems, Math. Comput. Simulation, 82 (2012), pp. 1894-1907. 
[25] G. KARYPIS AND V. KUMAR, A fast and high quality multilevel scheme for partitioning irregular graphs, SIAM J. Sci. Comput., 20 (1998), pp. 359-392.

[26] H. H. KIM, E. CHUNG, AND J. WANG, BDDC and FETI-DP preconditioners with adaptive coarse spaces for three-dimensional elliptic problems with oscillatory and high contrast coefficients, J. Comput. Phys., 349 (2017), pp. 191-214.

[27] H. H. KIM AND E. T. CHUNG, A BDDC algorithm with enriched coarse spaces for two-dimensional elliptic problems with oscillatory and high contrast coefficients, Multiscale Model. Simul., 13 (2015), pp. 571593.

[28] A. Klawonn, M. KÜHn, And O. RheInBaCh, Adaptive coarse spaces for FETI-DP in three dimensions, SIAM J. Sci. Comput., 38 (2016), pp. A2880-A2911.

[29] - Adaptive coarse spaces for FETI-DP in three dimensions with applications to heterogeneous diffusion problems, in Domain Decomposition Methods in Science and Engineering XXIII, C.-O. Lee, X.-C. Cai, D. E. Keyes, H. H. Kim, A. Klawonn, E.-J. Park, O. B. Widlund, eds., vol. 116 of Lect. Notes Comput. Sci. Eng, Springer, Cham, 2017, pp. 187-196.

[30] _ - FETI-DP and BDDC methods with a transformation of basis for heterogeneous problems: Connections to deflation, Tech. Report 01/2017, Fakultät für Mathematik und Informatik, TU Freiberg, Freiberg, 2017. http://tu-freiberg.de/fakult1/forschung/preprints

[31] A. Klawonn, M. Lanser, P. Radtke, And O. Rheinbach, On an adaptive coarse space and on nonlinear domain decomposition, in Domain Decomposition Methods in Science and Engineering XXI, J. Erhel, M. J. Gander, L. Halpern, G. Pichot, T. Sassi, O. B. Widlund, eds., vol. 98 of Lect. Notes Comput. Sci. Eng., Springer, Cham, 2014, pp. 71-83.

[32] A. KLAWONn, M. LANSER, AND O. RHEINBACH, Toward extremely scalable nonlinear domain decomposition methods for elliptic partial differential equations, SIAM J. Sci. Comput., 37 (2015), pp. C667-C696.

[33] A. KLAWONN, P. RADTKE, AND O. RHEINBACH, FETI-DP methods with an adaptive coarse space, SIAM J. Numer. Anal., 53 (2015), pp. 297-320.

[34] _ - A comparison of adaptive coarse spaces for iterative substructuring in two dimensions, Electron. Trans. Numer. Anal., 45 (2016), pp. 75-106. http://etna.ricam.oeaw.ac.at/vol.45.2016/pp75-106.dir/pp75-106.pdf

[35] A. KLAWONN AND O. RHEINBACH, A parallel implementation of dual-primal FETI methods for threedimensional linear elasticity using a transformation of basis, SIAM J. Sci. Comput., 28 (2006), pp. 18861906.

[36] - Inexact FETI-DP methods, Internat. J. Numer. Methods Engrg., 69 (2007), pp. 284-307.

[37] _ Robust FETI-DP methods for heterogeneous three dimensional elasticity problems, Comput. Methods Appl. Mech. Engrg., 196 (2007), pp. 1400-1414.

[38] _ Deflation, projector preconditioning, and balancing in iterative substructuring methods: connections and new results, SIAM J. Sci. Comput., 34 (2012), pp. A459-A484.

[39] A. KLAWONN AND O. B. WIDLUND, FETI and Neumann-Neumann iterative substructuring methods: connections and new results, Comm. Pure Appl. Math., 54 (2001), pp. 57-90.

[40] - Dual-primal FETI methods for linear elasticity, Comm. Pure Appl. Math., 59 (2006), pp. 1523-1572.

[41] A. KLAWOnn, O. B. WIDLUnd, AND M. DRYJA, Dual-primal FETI methods for three-dimensional elliptic problems with heterogeneous coefficients, SIAM J. Numer. Anal., 40 (2002), pp. 159-179.

[42] A. V. KNYAZEV, lobpcg.m, MATLAB implementation of the locally optimal block preconditioned conjugate gradient method, accessed: 2015-12-09.

https://www. mathworks.com/matlabcentral/fileexchange/48-lobpcg-m

[43] - Toward the optimal preconditioned eigensolver: locally optimal block preconditioned conjugate gradient method, SIAM J. Sci. Comput., 23 (2001), pp. 517-541.

[44] M. LANSER, Nonlinear FETI-DP and BDDC Methods, PhD. Thesis, Math. Nat. Fakultät, Universität zu Köln, 2015.

[45] J. LI AND O. B. WIDLUND, FETI-DP, BDDC, and block Cholesky methods, Internat. J. Numer. Methods Engrg., 66 (2006), pp. 250-271.

[46] J. Mandel, C. R. Dohrmann, And R. Tezaur, An algebraic theory for primal and dual substructuring methods by constraints, Appl. Numer. Math., 54 (2005), pp. 167-193.

[47] J. MANDEL AND B. SousedíK, Adaptive selection of face coarse degrees of freedom in the BDDC and the FETI-DP iterative substructuring methods, Comput. Methods Appl. Mech. Engrg., 196 (2007), pp. 1389-1399.

[48] J. MANDEL, B. SousedíK, AND J. ŠísteK, Adaptive BDDC in three dimensions, Math. Comput. Simulation, 82 (2012), pp. 1812-1831.

[49] T. P. A. Mathew, Domain Decomposition Methods for the Numerical Solution of Partial Differential Equations, Springer, Berlin, 2008.

[50] R. NABBEN AND C. VUIK, A comparison of deflation and coarse grid correction applied to porous media flow, SIAM J. Numer. Anal., 42 (2004), pp. 1631-1647. 
[51] — - A comparison of deflation and the balancing preconditioner, SIAM J. Sci. Comput., 27 (2006), pp. $1742-1759$.

[52] D.-S. OH, O. B. Widlund, S. Zampini, And C. R. Dohrmann, BDDC Algorithms with deluxe scaling and adaptive selection of primal constraints for Raviart-Thomas vector fields, Math. Comp., 87 (2018), pp. 659-692.

[53] C. PEChSTEIN AND C. R. Dohrmann, A unified framework for adaptive BDDC, Electron. Trans. Numer. Anal., 46 (2017), pp. 273-336.

http://etna.ricam.oeaw.ac.at/vol.46.2017/pp273-336.dir/pp273-336.pdf

[54] C. PEChSTEIN AND R. SCHEICHL, Analysis of FETI methods for multiscale PDEs. Part II: interface variation, Numer. Math., 118 (2011), pp. 485-529.

[55] D. J. RIXEN AND C. FARHAT, A simple and efficient extension of a class of substructure based preconditioners to heterogeneous structural mechanics problems, Internat. J. Numer. Methods Engrg., 44 (1999), pp. 489516.

[56] J. E. Roman, C. CAmpos, E. Romero, AND A. Tomas, SLEPc users manual, Tech. Rep. DSIC-II/24/02Revision 3.8, D. Sistemes Informàtics i Computació, Universitat Politècnica de València, Valencia, 2017.

[57] M. V. Sarkis Martins, Schwarz Preconditioners for Elliptic Problems with Discontinuous Coefficients using Conforming and Non-Conforming Elements, PhD. Thesis, Courant Institute of Mathematical Sciences, New York University, New York, 1994

[58] O. SCHENK, K. GÄRTNER, AND W. FICHTNER, Efficient sparse LU factorization with left-right looking strategy on shared memory multiprocessors, BIT, 40 (2000), pp. 158-176.

[59] B. Smith, P. BJøRstad, AND W. Gropp, Domain Decomposition: Parallel Multilevel Methods for Elliptic Partial Differential Equations, Cambridge University Press, Cambridge, 1996.

[60] N. Spillane, V. Dolean, P. Hauret, F. Nataf, C. Pechstein, And R. Scheichl, Abstract robust coarse spaces for systems of PDEs via generalized eigenproblems in the overlaps, Numer. Math., 126 (2014), pp. 741-770.

[61] N. SPILlANE AND D. J. RiXEN, Automatic spectral coarse spaces for robust finite element tearing and interconnecting and balanced domain decomposition algorithms, Internat. J. Numer. Methods Engrg., 95 (2013), pp. 953-990.

[62] A. Toselli And O. B. Widlund, Domain Decomposition Methods-Algorithms and Theory, Springer, Berlin, 2005. 